\title{
Vertebrate faUnA Of the Chambal River BASIN, With eMPHASIS ON THE NATIONAL CHAMBal SANCTUARY, INDIA
}

\section{Tarun Nair ${ }^{1} \&$ Y. Chaitanya Krishna ${ }^{2}$}

${ }^{1}$ Gharial Conservation Alliance, Centre for Herpetology - Madras Crocodile Bank Trust, P.O. Box 4, Mamallapuram, Tamil Nadu 603104, India

${ }_{1,2}$ Post-graduate Program in Wildlife Biology and Conservation, Wildlife Conservation Society - India Program, National

Centre for Biological Sciences, Bengaluru, Karnataka 560065, India; and Centre for Wildlife Studies, Bengaluru, Karnataka 560070, India

${ }^{2}$ Centre for Ecological Sciences, Indian Institute of Science, Malleshwaram, Bengaluru, Karnataka 560012, India

${ }^{2}$ Department of Ecology and Evolutionary Biology, Princeton University, Princeton, New Jersey 08544, USA

${ }^{1}$ tarunnair1982@gmail.com (corresponding author), ${ }^{2}$ chethikrishna@yahoo.com

Abstract: This research provides an updated checklist of vertebrate fauna of the Chambal River Basin in north-central India with an emphasis on the National Chambal Sanctuary. The checklist consolidates information from field surveys and a review of literature pertaining to this region. A total of 147 fish (32 families), 56 reptile (19 families), 308 bird (64 families) and 60 mammal (27 families) species are reported, including six Critically Endangered, 12 Endangered and 18 Vulnerable species, as categorised by the IUCN Red List of Threatened Species. This represents the first such extensive checklist for this region and provides an initial baseline of species for future research in this area.

Keywords: Checklist, Gharial, Important Bird Area, Red-crowned Roofed Turtle.

Abbreviation: IUCN - International Union for Conservation of Nature

Hindi Abstract: यह अनुसंधान उत्तर-मध्य भारत की चंबल नदी, खासकर राष्ट्रीय चंबल अभयारण्य के कशेरुकी जीवों कि एक जाँच सूचि प्रदान करता है। इस जाँच सूचि में चंबल क्षेत्र का साहित्यिक अवलोकन एवं क्षेत्र सर्वेक्षण की जानकारी भी समयोजित है। यहाँ मछूली कि कुल १४७ प्रजातियाँ (३२ परिवार), सरीसुप की कुल ५६ प्रजातियाँ ( $९ ९$ परिवार), पक्षी की कुल ३०८ प्रजातियाँ (६४ परिवार) और स्तनपायी जीवों की कुल ६० प्रजातियाँ (२९ परिवार) पायी जाती हैं, जिसमें कि आइ .यू .सी .एन. रेड लिस्ट ऑफ़ श्रेटंड स्पिसीस के अंतर्गत आने वाली ६ क्रिटिकली एनडेनजर्ड, १ २ एनडेनजर्ड और १८ वलनरेबल प्रजातियाँ हैं। यह इस क्षेत्र की पहली ऐसी व्यापक जाँच सूची है जो की भविष्य के लिए प्रजातियों के षोध की अधारसुची का प्रतिनिधित्व करती है।

DOI: http://dx.doi.org/10.11609/JoTT.03238.3620-41 | ZooBank: urn:Isid:zoobank.org:pub:F7DB75FF-1D30-4FDE-B73B-A4B0EE36CA56

Manuscript details: Ms \# 03238 | Received 18 June 2012 | Final received 01 January 2013 | Finally accepted 05 February 2013

Citation: Nair, T. \& Y.C. Krishna (2013). Vertebrate fauna of the Chambal River Basin, with emphasis on the National Chambal Sanctuary, India. Journal of Threatened Taxa 5(2): 3620-3641; doi:10.11609/JoTT.03238.3620-41

Copyright: (c) Nair \& Krishna 2013. Creative Commons Attribution 3.0 Unported License. JoTT allows unrestricted use of this article in any medium, reproduction and distribution by providing adequate credit to the authors and the source of publication.

Funding: The Centre for Herpetology - Madras Crocodile Bank Trust - Herpetological Conservation Research Fund for financial support during the compilation of this checklist. Tarun Nair was supported by the Department of Science and Technology, Government of India, National Centre for Biological Sciences, and Wildlife Conservation Society - India Program for field work in 2010. Chaitanya Krishna is currently supported by a Fulbright-Nehru Doctoral and Professional Research Fellowship.

Competing Interest: None.

Author Contribution: TN conducted fieldwork and secured funding. CK and TN jointly contributed towards reviewing literature and writing

Acknowledgements: We thank the Rajasthan, Madhya Pradesh and Uttar Pradesh Forest Departments for research permissions. We are grateful to Rakesh Vyas, R.S. Tomar, Banwari and Jyoti for providing logistical support for field work between 2006 and 2008; Rajeev Tomar for logistical support in 2010; Robin Kurian Abraham and Vidyadhar Atkore for reviewing the final version of the fish checklist and Nachiket Kelkar for comments on an early draft of the fish checklist; Rakesh Vyas, R.S. Tomar and Sunil Singhal for generously sharing their work-in-preparation; Archana Bali and Neelesh Dahanukar for providing reference material; and Divya Karnad for reviewing the manuscript. We thank two anonymous reviewers for their comments which improved the manuscript. We thank Saniya Chaplod for help with the Hindi abstract.

Author Details: TARUN NAIR is a conservation biologist with an interest in crocodilians, freshwater systems and human-wildlife conflict. Y. CHAITANYA KRISHNA is a grassland ecologist, having worked on four horned antelopes, pallid harriers, blackbuck and human-wildlife conflict.
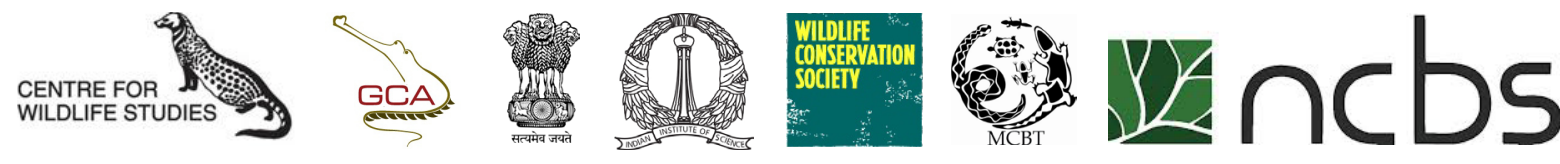


\section{INTRODUCTION}

Biodiversity inventories or checklists serve as repositories of baseline information on species occurrences, biogeography and their conservation status (Chandra \& Gajbe 2005). They are essential tools for developing our knowledge and understanding of biodiversity, and often the first step to undertake effective conservation action. This information is also fundamental to assess changes in species composition and distribution (Abraham et al. 2011) in the face of perturbations that may be anthropogenic (dams, mining, etc.) or natural (earthquakes, volcanoes, etc.).

Lying between $24^{\circ} 55^{\prime}-26^{\circ} 50^{\prime} \mathrm{N} \& 7^{\circ} 34^{\prime}-79^{\circ} 18^{\prime} \mathrm{E}$ (Fig. 1), the National Chambal Sanctuary (hereafter, NCS), was established between 1978 and 1983 by the states of Rajasthan, Madhya Pradesh and Uttar Pradesh to conserve the Gharial and the unique Chambal ecosystem. It covers nearly $1800 \mathrm{~km}^{2}$ across the three states, to form the first and only tri-state protected area in India. Despite being one of the last remnant rivers in the greater Gangetic Drainage Basin to have retained significant conservation values (Hussain \& Badola 2001), the Chambal River faces severe extractive and intrusive pressures for resources. The NCS is an Important Bird Area - Site Code IN-UP-11 and IN-RJ-11 (Islam \&
Rahmani 2004). A comprehensive database of species occurring in this landscape does not exist. Currently, this information is scattered throughout literature (Dubey \& Mehra 1959; Sale 1982; Sharma et al. 1995; Chandra \& Gajbe 2005; Saksena 2007; Sharma \& Choudhary 2007; Srivastava 2007; Tigerwatch 2008, 2009; Vyas et al. in prep.), difficult to procure and inaccessible to the general public or administration.

The aim of this paper is to compile information from several sources including peer-reviewed publications, reports and our field observations, in order to highlight the vertebrate faunal diversity (fishes, reptiles, birds and mammals), and provide a baseline, reference checklist for the region. We also discuss threats to the region's biodiversity, particularly in NCS.

\section{MATERIALS AND METHODS}

\section{Study area}

The Chambal Basin $\left(22^{\circ} 27^{\prime} \mathrm{N}-73^{\circ} 20^{\prime} \mathrm{E} \quad \&\right.$ $27^{\circ} 20^{\prime} \mathrm{N}-79^{\circ} 15^{\prime} \mathrm{E}$ ) is a rain-fed catchment and drains a total area of $143,219 \mathrm{~km}^{2}$ and is characterised by an undulating floodplain, gullies, forests, ravines, and a mosaic of land-use types (Hussain \& Badola 2001; Gopal \& Srivastava 2008). It is bound on the south, east

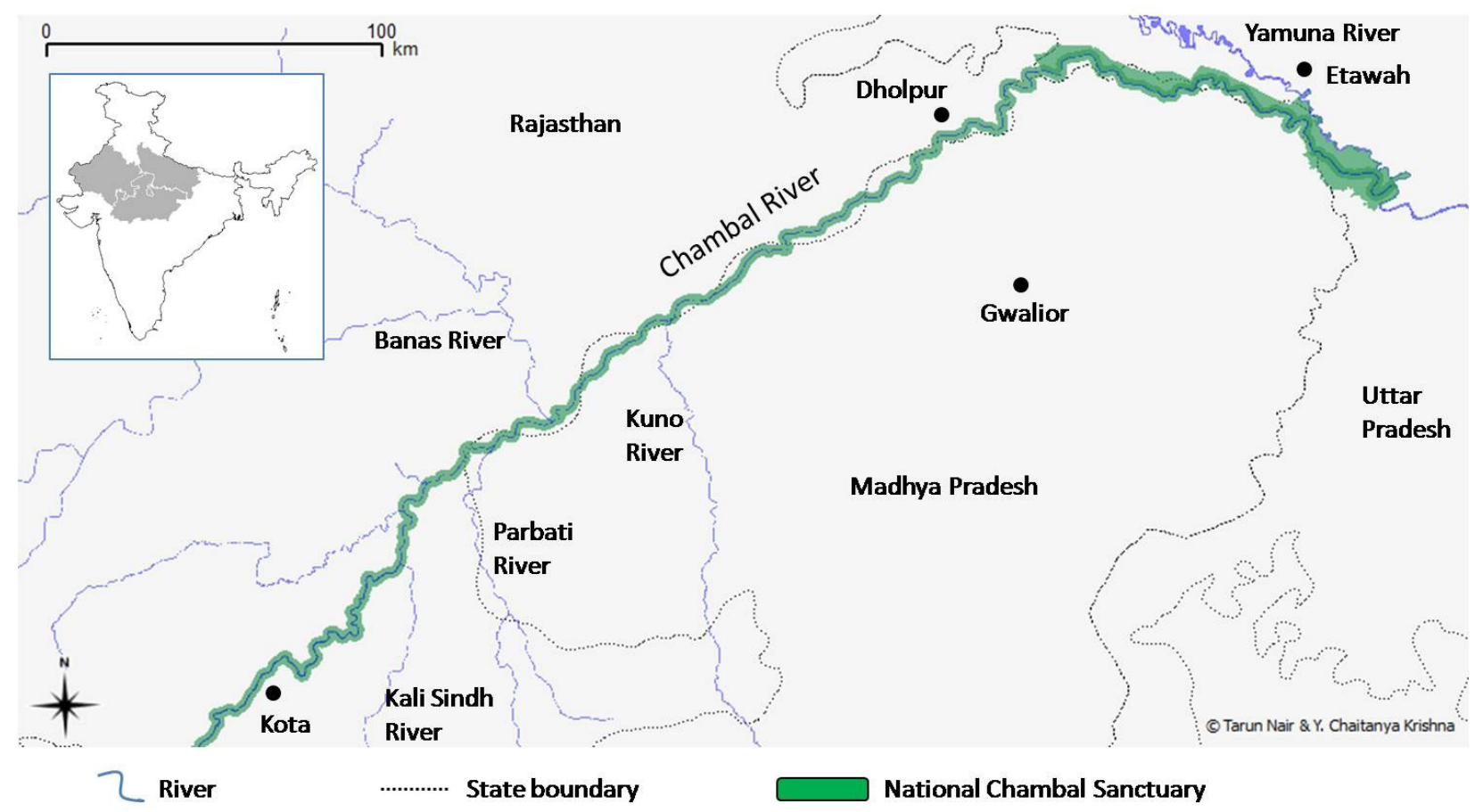

Figure 1. Location of the study area (Inset) across the states of Rajasthan, Madhya Pradesh and Uttar Pradesh; and map of the National Chambal Sanctuary, covering an area of approximately $1800 \mathrm{~km}^{2}$. 
and west by the Vindhyan mountain range and on the north-west by the Aravallis. The $960 \mathrm{~km}$ long Chambal River originates in the northern slopes of the Vindhyan escarpment and joins the Yamuna River near Bareh in Uttar Pradesh. The tributaries of the Chambal include Shipra, Choti Kalisindh, Sivanna, Retam, Ansar, Kali Sindh, Banas, Parbati, Seep, Kuwari, Kuno, Alnia, Mej, Chakan, Parwati, Chamla, Gambhir, Lakhunder, Khan, Bangeri, Kedel and Teelar (Jain et al. 2007; Gopal \& Srivastava 2008). The NCS consists of a $\sim 600 \mathrm{~km}$ long arc of the Chambal River. Over this arc, two stretches of the Chambal are protected as the National Chambal Sanctuary - the upper sector, extending from Jawahar Sagar Dam to Kota Barrage, and the lower sector, extending from Keshoraipatan in Rajasthan to the Chambal-Yamuna confluence in Uttar Pradesh.

The NCS lies within the semi-arid zone of northwestern India at the border of Madhya Pradesh, Rajasthan and Uttar Pradesh States (Hussain 1999). Ambient air temperatures range from $2-49{ }^{\circ} \mathrm{C}$ (Tarun Nair 20092010 pers. obs.) with a mean annual precipitation of $590 \mathrm{~mm}$, the bulk of which is received during the southwest monsoon (Hussain 1999, 2009). From the source down to its confluence with the Yamuna, the Chambal has a fall of about $732 \mathrm{~m}$. The Chambal averages $400 \mathrm{~m}$ in width while depth ranges from 1-26 m (Hussain 1991).

The vegetation is classified as ravine and thorn forest (Champion \& Seth 1968). Evergreen riparian vegetation is completely absent, with only sparse ground-cover along the severely eroded river banks and adjacent ravine lands (Hussain 1999, 2009). The region was also subject to intentional aerial seeding of Prosopis juliflora in the 1980s, as a ravine reclamation measure (Prasad 1988), and as a consequence $P$. juliflora is widespread in the region.

Much of the basin has been influenced by a long history of human occupation (Kaul 1962). Anthropogenic influences are chiefly in the form of sand-mining; bankside cultivation; domestic activities like bathing, washing and water collection; fishing; poaching; livestock herding; grass-soaking; river crossing and temple fairs. The Chambal River also suffers severe hydrological modifications from water impoundment and extraction.

\section{Data compilation and collection}

Keywords such as Chambal, checklist, inventory, biodiversity, mammals, birds, reptiles, fish, fauna and occurrence were used in several variations and combinations in Google, Google Scholar, PubMed and Science Direct. The references within the resulting documents were also sourced and reviewed. Similarly, five of the most widely recognised databases of published literature on Indian biodiversity, namely, Indian Forester, Journal of the Bombay Natural History Society, Zoos' Print Journal, Conservation Assessment and Management Plan (C.A.M.P.) reports and Journal of Threatened Taxa, were also reviewed for relevant information. Preliminary checklists of fishes, reptiles, birds and mammals were prepared based on a review of published literature (Dubey \& Mehra 1959; Sharma et al. 1995; Chauhan \& Narain 2001; Sivakumar 2002; Sharma 2003; Khudsar 2004; Sundar 2004; Vyas 2004; Vyas \& Singh 2004; Chandra \& Gajbe 2005; Nair 2009), survey reports (Tigerwatch 2008, 2009; Vyas et al. in prep.), status reports and taxonomic assessments (Sale 1982; Molur \& Walker 1998a,b; Rao 1988; Islam \& Rahmani 2002; Molur et al. 2002; Islam \& Rahmani 2004; Molur et al. 2005; Schätti \& Schmitz 2006; Saksena 2007; Sharma \& Choudhary 2007; Srivastava 2007; Choudhury et al. 2008; Sanderson et al. 2008; Driscoll \& Nowell 2009; Böhm \& Richman 2010; Das et al. 2010; Murphy \& Lobo 2010; Tenzin 2010; Vishwanath 2010a,b; Vidthayanon et al. 2011; BirdLife International 2012a,b).

Opportunistic field observations were made during field surveys in March-April 2006, February 2008, October 2009 and from December 2009 to May 2010, while collecting information on human-crocodile conflict, gharial habitat-use and population estimation.

We validated species checklists based on available ecological knowledge and distributional records for each species. For instance, Sale (1982) reports the presence of Varanus salvator (Common Water Monitor) in the NCS. However, in India, V. salvator is reported to be restricted to the eastern and northeastern states of mainland India (Whitaker \& Whitaker 1980; Molur \& Walker 1998b; Bennett et al. 2010), and is hence omitted from our checklist.

The taxonomic classification, nomenclature and sequence followed Eschmeyer (2012) and Eschmeyer \& Fong (2012) for fishes; Turtle Taxonomy Working Group [van Dijk, P.P., J.B. Iverson, H.B. Shaffer, R. Bour \& A.G.J. Rhodin] (2011) for turtles and tortoises; ITIS (2012) for other reptiles; BirdLife International (2012) for birds; and Wilson \& Reeder (2005) for mammals.

\section{RESULTS AND DISCUSSION}

Faunal diversity: We recorded 147 fish species comprising 32 families (Table 2), 56 reptile species comprising 19 families (Table 3), 308 bird species comprising 64 families (Table 4) and 60 mammal species 
comprising 27 families (Table 5) from this region, based on available literature and our field observations. This includes six Critically Endangered, 12 Endangered and 18 Vulnerable species (see Table 1 ), as categorised by the IUCN Red List of Threatened Species (IUCN 2011).

The NCS is among the most important and significant habitats where several globally threatened fauna still survive. Apart from being a strong candidate for World Heritage and Ramsar Convention listings, the NCS is also subject to international treaties like the Convention on the Conservation of Migratory Species of Wild Animals (Bonn Convention), which lists both flagship species of the NCS - the Gharial Gavialis gangeticus and Gangetic River Dolphins Platanista gangetica. It contains the most viable breeding populations of the Critically Endangered Gharial and Red-crowned Roofed Turtle Batagur kachuga. It is also among the most important strongholds of the Deccan Mahaseer Tor khudree, Putitor Mahaseer Tor putitora, Narrow-headed Softshell Turtle Chitra indica, Three-striped Roofed Turtle Batagur dhongoka, Indian Skimmer Rynchops albicollis, Black-bellied Tern Sterna acuticauda, Sarus Crane Grus antigone and Gangetic River Dolphin Platanista gangetica. The NCS functions as a vital source and nursery for fish fry and fingerlings, contributing significantly to downstream fisheries in the Gangetic river system (Sivakumar \& Choudhury 2008). It is an Important Bird Area particularly for the Oriental White-backed Vulture Gyps bengalensis, Longbilled Vulture Gyps indicus, Pallas's Fish-Eagle Haliaeetus leucoryphus and Greater Spotted Eagle Aquila clanga among others (Islam \& Rahmani 2004). The NCS also serves as among the best over-wintering sites for migratory birds. In addition, this river sanctuary also forms a vital corridor and link for the movement and dispersal of Tigers Panthera tigris from the source population of Ranthambore Tiger Reserve to the protected areas of Kuno-Palpur, Madhav National Park and Darrah-Mukundra (Reddy et al. 2012; Rakesh Vyas February 2008 pers. comm.).

Threats: The Chambal faces severe extractive and intrusive pressures in the form of water impoundment and abstraction, sand- and stone-mining, fishing, poaching, riparian agriculture, livestock grazing, firewood collection, miscellaneous domestic activities, and infrastructural development (Hussain 2009; Nair 2010; Katdare et al. 2011; MoEF 2011; Tarun Nair 2006, 2008, 2009-2013 pers. obs.).

Seven major, 12 medium and 134 minor irrigation projects operating in the Chambal River Basin, have greatly reduced river flow (Hussain \& Badola 2001). Misleading environment impact assessments have
Table 1. List of species from the Chambal River Basin in the IUCN Red List of Threatened Species (IUCN 2011)

\begin{tabular}{|c|c|c|c|}
\hline & $\begin{array}{l}\text { Critically } \\
\text { Endangered }\end{array}$ & Endangered & Vulnerable \\
\hline \multirow[t]{5}{*}{ Fishes } & & $\begin{array}{l}\text { Himantura } \\
\text { chaophraya* }\end{array}$ & Botia rostrata \\
\hline & & Puntius arulius* & \\
\hline & & Tor khudree & \\
\hline & & Tor putitora & \\
\hline & & Clarias magur* & \\
\hline \multirow[t]{4}{*}{ Reptiles } & $\begin{array}{l}\text { Gavialis } \\
\text { gangeticus }\end{array}$ & $\begin{array}{l}\text { Batagur } \\
\text { dhongoka }\end{array}$ & $\begin{array}{l}\text { Crocodylus } \\
\text { palustris }\end{array}$ \\
\hline & Batagur kachuga & Chitra indica & Hardella thurjii \\
\hline & & & $\begin{array}{l}\text { Nilssonia } \\
\text { gangetica }\end{array}$ \\
\hline & & & Nilssonia hurum \\
\hline \multirow[t]{8}{*}{ Birds } & Gyps bengalensis & $\begin{array}{l}\text { Neophron } \\
\text { percnopterus }\end{array}$ & $\begin{array}{l}\text { Leptoptilos } \\
\text { javanicus }\end{array}$ \\
\hline & Gyps indicus & & Pelecanus crispus \\
\hline & Gyps tenuirostris & & $\begin{array}{l}\text { Haliaeetus } \\
\text { leucoryphus }\end{array}$ \\
\hline & Sarcogyps calvus & & Aquila clanga \\
\hline & & & Aquila heliaca \\
\hline & & & Grus antigone \\
\hline & & & $\begin{array}{l}\text { Rynchops } \\
\text { albicollis }\end{array}$ \\
\hline & & & $\begin{array}{l}\text { Amandava } \\
\text { formosa }\end{array}$ \\
\hline \multirow[t]{5}{*}{ Mammals } & & $\begin{array}{l}\text { Prionailurus } \\
\text { viverrinus }\end{array}$ & Melursus ursinus \\
\hline & & Panthera tigris & $\begin{array}{l}\text { Lutrogale } \\
\text { perspicillata }\end{array}$ \\
\hline & & Cuon alpinus & Gazella gazella \\
\hline & & $\begin{array}{l}\text { Platanista } \\
\text { gangetica }\end{array}$ & $\begin{array}{l}\text { Tetracerus } \\
\text { quadricornis }\end{array}$ \\
\hline & & & Rusa unicolor \\
\hline
\end{tabular}

*See endnote (fishes), after Table 2 for more details.

permitted recently commissioned water abstraction projects to operate in the NCS by suppressing information on species' occurrences and falsely stating "As there is no significant flora and fauna in or around Chambal River, there should also not be any ecological impacts from the increase in abstraction" (RUSDIP 2008, page 44). Up- and downstream effects of dams are well-known, stemming from inundation, flow manipulation, and fragmentation. Dams obstruct the dispersal and migration of organisms, and these and other effects have been directly linked to loss of populations and entire species of freshwater fish (Nilsson et al. 2005). Low-flows in the Chambal River result in discontinuity between deep pools in the 
Table 2. Checklist of fishes of the Chambal River Basin

\begin{tabular}{|c|c|c|c|c|c|c|c|}
\hline & Species & $\begin{array}{c}\text { IUCN } \\
\text { status } \\
(2009- \\
2011)\end{array}$ & References & & Species & $\begin{array}{c}\text { IUCN } \\
\text { status } \\
(2009- \\
2011)\end{array}$ & References \\
\hline & Dasyatidae & & & 34 & Esomus danricus (Hamilton, 1822) & LC & $A, C, E, G$ \\
\hline 1 & Himantura marginata ${ }^{1}$ (Blyth, 1860) & DD & A & 35 & Garra gotyla (Gray, 1830) & LC & $C, E, G, H$ \\
\hline \multirow{2}{*}{2} & \multirow{2}{*}{$\begin{array}{l}\text { Himantura chaophraya }{ }^{2} \text { Monkolprasit \& } \\
\text { Roberts, } 1990\left[\text { H. polylepis }{ }^{2^{*}} \text { (Bleeker, }\right. \\
\text { 1852)] }\end{array}$} & \multirow{2}{*}{ EN } & \multirow{2}{*}{$\mathrm{F}$} & 36 & Garra lamta (Hamilton, 1822) & LC & $A, G$ \\
\hline & & & & 37 & Garra mullya (Sykes, 1839) & LC & $E, G$ \\
\hline \multirow[t]{2}{*}{3} & Pastinachus sephen (Forsskål, 1775) & DD & $\mathrm{C}, \mathrm{F}$ & 38 & $\begin{array}{l}\text { Hypophthalmichthys } \\
\text { molitrix }^{\text {INT }} \text { (Valenciennes, 1844) }\end{array}$ & - & $C, E, G$ \\
\hline & Notopteridae & & & \multirow{2}{*}{39} & \multirow{2}{*}{$\begin{array}{l}\text { Hypophthalmichthys } \\
\text { nobilis (Richardson, 1845) }\end{array}$} & \multirow{2}{*}{-} & \multirow{2}{*}{$E, G$} \\
\hline 4 & Chitala chitala (Hamilton, 1822) & NT & $A, B, C, D, E, G$ & & & & \\
\hline \multirow[t]{2}{*}{5} & Notopterus notopterus (Pallas, 1769) & LC & $A, C, D, E, G, H$ & 40 & Labeo angra (Hamilton, 1822) & LC & $E, G$ \\
\hline & Anguillidae & & & 41 & Labeo bata (Hamilton, 1822) & LC & $A, C, D, E, G, H$ \\
\hline \multirow[t]{2}{*}{6} & Anguilla bengalensis (Gray, 1831) & LC & C & 42 & Labeo boga (Hamilton, 1822) & LC & $A, C, E, G$ \\
\hline & Clupeidae & & & 43 & Labeo boggut (Sykes, 1839) & LC & $A, C, E, G, H$ \\
\hline 7 & Gonialosa manmina (Hamilton, 1822) & LC & J & 44 & Labeo calbasu (Hamilton, 1822) & LC & $A, C, E, G, H$ \\
\hline 8 & Gudusia chapra (Hamilton, 1822) & LC & $A, C, D, E, G, I$ & 45 & Labeo dyocheilus (McClelland, 1839) & LC & $A, C, D, E, G$ \\
\hline \multirow[t]{2}{*}{9} & Tenualosa ilisha (Hamilton, 1822) & - & $A, C, D$ & 46 & Labeo fimbriatus (Bloch, 1795) & LC & $\mathrm{C}, \mathrm{E}, \mathrm{G}$ \\
\hline & Cyprinidae & & & 47 & Labeo gonius (Hamilton, 1822) & LC & $A, C, D, E, H$ \\
\hline \multirow{2}{*}{10} & \multirow{2}{*}{$\begin{array}{l}\text { Amblypharyngodon microlepis (Bleeker, } \\
\text { 1853) }\end{array}$} & \multirow{2}{*}{ LC } & \multirow{2}{*}{ G } & 48 & Labeo pangusia (Hamilton, 1822) & NT & E \\
\hline & & & & \multirow{2}{*}{49} & \multirow{2}{*}{$\begin{array}{l}\text { Labeo rajasthanicus Datta \& Majumdar, } \\
1970\end{array}$} & & \\
\hline \multirow[t]{2}{*}{11} & \multirow{2}{*}{$\begin{array}{l}\text { Amblypharyngodon mola (Hamilton, } \\
1822 \text { ) }\end{array}$} & \multirow{2}{*}{ LC } & \multirow{2}{*}{$A, C, E, G$} & & & - & E \\
\hline & & & & 50 & Labeo rohita (Hamilton, 1822) & LC & $A, C, E, G, H, I$ \\
\hline 12 & Aspidoparia jaya (Hamilton, 1822) & LC & J & 51 & Laubuca laubuca (Hamilton, 1822) & LC & $\mathrm{A}, \mathrm{C}, \mathrm{H}$ \\
\hline 13 & Aspidoparia morar (Hamilton, 1822) & LC & $A, E, G$ & 52 & Osteobrama cotio (Hamilton, 1822) & LC & $A, C, D, E, G, H$ \\
\hline 14 & Bangana dero (Hamilton, 1822) & LC & A & 53 & Puntius amphibius (Valenciennes, 1842) & DD & $E, G$ \\
\hline 15 & Barilius barila (Hamilton, 1822) & LC & $A, G, H$ & 54 & Puntius arulius ${ }^{5}$ (Jerdon, 1849) & EN & G \\
\hline 16 & Barilius bendelisis (Hamilton, 1807) & LC & $\mathrm{A}, \mathrm{C}, \mathrm{G}, \mathrm{H}, \mathrm{I}$ & 55 & Puntius chola (Hamilton, 1822) & LC & $E, G$ \\
\hline 17 & Barilius modestus Day, 1872 & - & G & 56 & Pethia conchonius (Hamilton, 1822) & LC & $A, C$ \\
\hline 18 & Barilius shacra (Hamilton, 1822) & LC & B & 57 & Puntius guganio (Hamilton, 1822) & LC & 1 \\
\hline 19 & Barilius vagra (Hamilton, 1822) & LC & G & 58 & Pethia phutunio (Hamilton, 1822) & LC & A \\
\hline 20 & $\begin{array}{l}\text { Bengala elanga (Hamilton, 1822) } \\
{\left[\text { Megarasbora elanga }^{3}\right]}\end{array}$ & LC & G & 59 & Systomus sarana (Hamilton, 1822) & LC & $A, C, D, E, G, H$ \\
\hline 21 & Opsarius barna (Hamilton, 1822) & LC & $\mathrm{C}, \mathrm{G}$ & 60 & Puntius sophore (Hamilton, 1822) & LC & $A, C, D, E, G, I$ \\
\hline 22 & Carassius carassius $^{\mathrm{INT}}$ (Linnaeus, 1758) & - & $\mathrm{C}, \mathrm{E}, \mathrm{G}$ & 61 & Pethia ticto (Hamilton, 1822) & LC & $A, C, D, E, G, H$ \\
\hline 23 & Catla catla (Hamilton, 1822) [Gibelion & LC & $A, B, C, E, G, H, I$ & 62 & Puntius vittatus Day, 1865 & LC & G \\
\hline & & & & 63 & Raiamas bola (Hamilton, 1822) & LC & $B, C, E, H$ \\
\hline 24 & Chagunius chagunio (Hamilton, 1822) & LC & $A, E, G$ & 64 & Rasbora daniconius (Hamilton, 1822) & LC & $A, C, D, E, H, I$ \\
\hline 25 & Chela cachius (Hamilton, 1822) & LC & $A, E, G$ & 65 & Rasbora rasbora (Hamilton, 1822) & LC & 1 \\
\hline $\begin{array}{l}26 \\
27\end{array}$ & $\begin{array}{l}\text { Cirrhinus mrigala (Hamilton, 1822) } \\
\text { Cirrhinus reba (Hamilton, 1822) }\end{array}$ & $\begin{array}{ll}\mathrm{LC} \\
\mathrm{LC}\end{array}$ & $\begin{array}{c}A, C, G, H, I \\
A, C, D, E, G, H\end{array}$ & 66 & Salmophasia bacaila (Hamilton, 1822) & LC & $A, B, C, D, G, H$ \\
\hline$\frac{21}{28}$ & Crossocheilus latius (Hamilton, 1822) & $L C$ & $\frac{A, C, D, E, U, n}{A, D, G}$ & 67 & Salmophasia balookee (Sykes, 1839) & LC & $\mathrm{A}, \mathrm{B}, \mathrm{C}, \mathrm{G}, \mathrm{H}, \mathrm{I}$ \\
\hline 20 & Ctenopharyngodon & 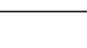 & 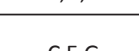 & 68 & Salmophasia phulo (Hamilton, 1822) & LC & G \\
\hline 29 & idella (Valenciennes, 1844) & - & $\mathrm{C}, \mathrm{E}, \mathrm{G}$ & 69 & Salmophasia punjabensis (Day, 1872) & - & G \\
\hline 30 & Cyprinus carpio ${ }^{\text {INT }}$ Linnaeus, 1758 & - & $C, D, E, G$ & 70 & Securicula gora (Hamilton, 1822) & LC & $A, E, G$ \\
\hline 31 & Danio rerio (Hamilton, 1822) & LC & $A, E, G$ & 71 & Tor khudree (Sykes, 1839) & EN & $E, G, K$ \\
\hline 32 & $\begin{array}{l}\text { Devario aequipinnatus (McClelland, } \\
\text { 1839) }\end{array}$ & LC & G & 72 & Tor tor (Hamilton, 1822) & NT & $A, C, D, E, G, H$ \\
\hline 33 & Devario devario (Hamilton, 1822) & LC & $A, C, E, G, H$ & 73 & Tor putitora (Hamilton, 1822) & EN & $C, G$ \\
\hline
\end{tabular}




\begin{tabular}{|c|c|c|c|}
\hline & Species & $\begin{array}{c}\text { IUCN } \\
\text { status } \\
(2009- \\
2011) \\
\end{array}$ & References \\
\hline & Psilorhynchidae & & \\
\hline \multirow[t]{2}{*}{74} & Psilorhynchus balitora (Hamilton, 1822) & LC & G \\
\hline & Cobitidae & & \\
\hline 75 & Botia birdi Chaudhuri, 1909 & - & $E, G$ \\
\hline 76 & Botia lohachata Chaudhuri, 1912 & - & $E, G$ \\
\hline 77 & Botia rostrata Günther, 1868 & VU & G \\
\hline \multirow[t]{2}{*}{78} & $\begin{array}{l}\text { Lepidocephalichthys guntea (Hamilton, } \\
\text { 1822) }\end{array}$ & LC & $A, S, E, G$ \\
\hline & Nemacheilidae & & \\
\hline 79 & Acanthocobitis botia (Hamilton, 1822) & LC & $\mathrm{A}, \mathrm{C}, \mathrm{E}, \mathrm{H}, \mathrm{I}$ \\
\hline 80 & Nemacheilus carletoni Fowler, 1924 & - & G \\
\hline 81 & Nemacheilus corica (Hamilton, 1822) & LC & G \\
\hline 82 & Nemacheilus denisoni Day, 1867 & LC & G \\
\hline 83 & $\begin{array}{l}\text { Paraschistura montana (McClelland, } \\
\text { 1838) }\end{array}$ & - & G \\
\hline 84 & $\begin{array}{l}\text { Schistura baluchiorum }{ }^{6} \text { (Zugmayer, } \\
\text { 1912) }\end{array}$ & - & G \\
\hline 85 & Schistura beavani (Günther 1868) & LC & A \\
\hline 86 & Schistura horai (Menon, 1952) & - & G \\
\hline \multirow[t]{2}{*}{87} & Schistura rupecula McClelland, 1838 & LC & G \\
\hline & Bagridae & & \\
\hline 88 & Mystus bleekeri (Day, 1877) & LC & $A, C, E, G$ \\
\hline 89 & Mystus cavasius (Hamilton, 1822) & LC & $A, C, E, G$ \\
\hline 90 & Mystus tengara (Hamilton, 1822) & & 1 \\
\hline 91 & Mystus vittatus (Bloch, 1794) & LC & $A, C, E, G$ \\
\hline 92 & Rita rita (Hamilton, 1822) & LC & $A, B, C, D, E, G, H, I$ \\
\hline 93 & Sperata aor (Hamilton, 1822) & LC & $A, C, D, E, G, H$ \\
\hline \multirow[t]{2}{*}{94} & Sperata seenghala (Sykes, 1839) & LC & $A, C, D, E, G, H$ \\
\hline & Siluridae & & \\
\hline 95 & Ompok bimaculatus (Bloch, 1794) & NT & $\mathrm{A}, \mathrm{C}, \mathrm{D}, \mathrm{E}, \mathrm{G}, \mathrm{H}, \mathrm{I}$ \\
\hline \multirow[t]{2}{*}{96} & Wallago attu (Bloch \& Schneider, 1801) & NT & $A, B, C, D, E, G, H, I$ \\
\hline & Schilbeidae & & \\
\hline 97 & Ailia coila (Hamilton, 1822) & NT & $E, G$ \\
\hline 98 & Ailiichthys punctata Day, 1872 & DD & B \\
\hline 99 & Clupisoma garua (Hamilton, 1822) & LC & $B, C, E, G$ \\
\hline 100 & Eutropiichthys murius (Hamilton, 1822) & LC & 1 \\
\hline 101 & Eutropiichthys vacha (Hamilton, 1822) & LC & $A, C, D, E, G, H, I$ \\
\hline 102 & Neotropius atherinoides (Bloch, 1794) & LC & 1 \\
\hline \multirow[t]{2}{*}{103} & Silonia silondia (Hamilton, 1822) & LC & $\mathrm{A}, \mathrm{C}, \mathrm{D}, \mathrm{E}, \mathrm{G}, \mathrm{H}, \mathrm{I}$ \\
\hline & Pangasiidae & & \\
\hline \multirow[t]{2}{*}{104} & Pangasius pangasius (Hamilton, 1822) & LC & $A, B, C, D$ \\
\hline & Amblycipitidae & & \\
\hline \multirow[t]{2}{*}{105} & Amblyceps mangois (Hamilton, 1822) & LC & G \\
\hline & Sisoridae & & \\
\hline 106 & Gogangra viridescens (Hamilton, 1822) & LC & A \\
\hline
\end{tabular}

\begin{tabular}{|c|c|c|c|}
\hline & Species & $\begin{array}{c}\text { IUCN } \\
\text { status } \\
(2009- \\
2011)\end{array}$ & References \\
\hline 107 & Bagarius bagarius (Hamilton, 1822) & NT & $A, C, D, E, G, H, I$ \\
\hline 108 & Bagarius yarrelli (Sykes, 1839) & NT & G \\
\hline 109 & Gagata cenia (Hamilton, 1822) & LC & G \\
\hline 110 & Gagata sexualis Tilak, 1970 & LC & 1 \\
\hline 111 & Glyptothorax botius (Hamilton, 1822) & LC & A \\
\hline 112 & $\begin{array}{l}\text { Glyptothorax } \\
\text { pectinopterus (McClelland, 1842) }\end{array}$ & LC & G \\
\hline 113 & Glyptothorax telchitta (Hamilton, 1822) & LC & G \\
\hline 114 & Nangra nangra (Hamilton, 1822) & LC & G \\
\hline \multirow[t]{2}{*}{115} & Sisor rabdophorus Hamilton, 1822 & LC & G \\
\hline & Erethistidae & & \\
\hline \multirow[t]{2}{*}{116} & Pseudolaguvia ribeiroi (Hora, 1921) & LC & 1 \\
\hline & Clariidae & & \\
\hline 117 & Clarias magur ${ }^{7}$ (Linnaeus, 1758) & EN & $C, D, E, G$ \\
\hline \multirow[t]{2}{*}{118} & Clarias gariepinus ${ }^{\mathrm{INT}}$ (Burchell, 1822) & - & $E, G$ \\
\hline & Heteropneustidae & & \\
\hline \multirow[t]{2}{*}{119} & Heteropneustes fossilis (Bloch, 1794) & LC & $A, C, D, E, G, I$ \\
\hline & Aplocheilidae & & \\
\hline 120 & Aplocheilus blockii Arnold, 1911 & LC & G \\
\hline \multirow[t]{2}{*}{121} & $\begin{array}{l}\text { Aplocheilus lineatus (Valenciennes, } \\
\text { 1846) }\end{array}$ & LC & G \\
\hline & Belonidae & & \\
\hline \multirow[t]{2}{*}{122} & Xenentodon cancila (Hamilton, 1822) & LC & $A, B, C, D, E, G, H, I$ \\
\hline & Adrianichthyidae & & \\
\hline \multirow[t]{2}{*}{123} & Oryzias melastigma (McClelland, 1839) & LC & G \\
\hline & Synbranchidae & & \\
\hline \multirow[t]{2}{*}{124} & Monopterus cuchia (Hamilton, 1822) & LC & $\mathrm{C}, \mathrm{E}, \mathrm{G}$ \\
\hline & Mastacembelidae & & \\
\hline 125 & $\begin{array}{l}\text { Macrognathus aral (Bloch \& Schneider, } \\
\text { 1801) }\end{array}$ & LC & G \\
\hline 126 & Macrognathus pancalus Hamilton, 1822 & LC & $A, C, D, G$ \\
\hline \multirow[t]{2}{*}{127} & $\begin{array}{l}\text { Mastacembelus armatus (Lacepède, } \\
\text { 1800) }\end{array}$ & LC & $A, C, D, G, H, I$ \\
\hline & Ambassidae & & \\
\hline 128 & Chanda nama Hamilton, 1822 & LC & $A, C, D, E, G, H, I$ \\
\hline 129 & Pseudambassis baculis (Hamilton, 1822) & LC & G \\
\hline \multirow[t]{2}{*}{130} & Pseudambassis ranga (Hamilton, 1822) & LC & $A, C, D, G, H$ \\
\hline & Badidae & & \\
\hline \multirow[t]{2}{*}{131} & Badis badis (Hamilton, 1822) & LC & A \\
\hline & Sciaenidae & & \\
\hline \multirow[t]{2}{*}{132} & Otolithoides pama ${ }^{8}$ (Hamilton, 1822) & - & A \\
\hline & Nandidae & & \\
\hline \multirow[t]{2}{*}{133} & Nandus nandus (Hamilton, 1822) & LC & $A, B, C, E, G, H$ \\
\hline & Mugilidae & & \\
\hline 134 & Mugil cephalus ${ }^{9}$ Linnaeus, 1758 & LC & G \\
\hline
\end{tabular}




\begin{tabular}{|c|c|c|c|}
\hline & Species & $\begin{array}{c}\text { IUCN } \\
\text { status } \\
(2009- \\
2011)\end{array}$ & References \\
\hline 135 & Rhinomugil corsula (Hamilton, 1822) & LC & $A, C, D, E, H, I$ \\
\hline \multirow[t]{2}{*}{136} & Sicamugil cascasia (Hamilton, 1822) & LC & G \\
\hline & Cichlidae & & \\
\hline \multirow[t]{2}{*}{137} & $\begin{array}{l}\text { Oreochromis mossambicus }{ }^{\mathrm{INT} T} \text { (Peters, } \\
\text { 1852) }\end{array}$ & - & $\mathrm{G}, \mathrm{I}$ \\
\hline & Gobiidae & & \\
\hline \multirow[t]{2}{*}{138} & Glossogobius giuris (Hamilton, 1822) & - & $A, C, E, G, H, I$ \\
\hline & Anabantidae & & \\
\hline \multirow[t]{2}{*}{139} & Anabas testudineus (Bloch, 1792) & DD & $E, G$ \\
\hline & Osphronemidae & & \\
\hline 140 & $\begin{array}{l}\text { Trichogaster fasciata Bloch \& Schneider, } \\
1801\end{array}$ & LC & $C, E, G$ \\
\hline 141 & Trichogaster labiosa Day, 1877 & - & G \\
\hline \multirow[t]{2}{*}{142} & Trichogaster lalius (Hamilton, 1822) & LC & G \\
\hline & Channidae & & \\
\hline 143 & Channa gachua (Hamilton, 1822) & LC & $A, C$ \\
\hline 144 & Channa marulius (Hamilton, 1822) & LC & $A, C, D, E, G, H$ \\
\hline 145 & $\begin{array}{l}\text { Channa orientalis }{ }^{10} \text { Bloch \& Schneider, } \\
1801\end{array}$ & - & $E, G$ \\
\hline 146 & Channa punctata (Bloch, 1793) & LC & $A, C, D, E, G, H, I$ \\
\hline 147 & Channa striata (Bloch, 1793) & LC & $A, C, D, E, G, H$ \\
\hline
\end{tabular}

${ }^{1}$ Himantura marginata is primarily a marine fish, occasionally entering brackish waters. We do not have information of the specimen or identification. This record needs to be verified.

${ }^{2}$ Himantura chaophraya is not known from India. We do not have information of the specimen or identification. This record needs to be verified.

${ }^{2 *} \mathrm{H}$. chaophraya is considered as a synonym of H. polylepis by the IUCN Red List (Vidthayanon et al. 2011)

${ }^{3}$ Bengala elanga is considered as Megarasbora elanga by the IUCN Red List (Vishwanath 2010b).

${ }^{4}$ Catla catla is considered as Gibelion catla by the IUCN Red List (Tenzin 2010). ${ }^{5}$ Dawkinsia arulius (reported earlier as Puntius arulius) is known only from South India (Knight et al. 2011). This species may be Dravidia melanampyx or $D$. fasciatus but its taxonomy is unresolved (Abraham 2011).

Also see Pethiyagoda et al. (2012) for species hitherto referred to the genus Puntius.

${ }^{6}$ Schistura baluchiorum is mainly distributed in the Indus drainage. $S$.

baluchiorum is listed here because of its doubtful synonymy with Noemacheilus rajasthanicus. However, if $N$. rajasthanicus is a valid species this record should refer to it.

${ }^{7}$ Clarias magur has been described as $C$. batrachus in previous reports. $C$. batrachus is now reported only from the Sunda Islands [see Ng \& Kottelat (2008), and Vishwanath (2010a) for details].

${ }^{8}$ Otolithoides pama is primarily an estuarine / marine fish. We do not have information of the specimen or identification. This record needs to be verified. ${ }^{9}$ Mugil cephalus is primarily a marine species, and is introduced in freshwater systems. We do not have information of the specimen or identification. This record needs to be verified.

${ }^{10}$ Channa orientalis is endemic to Sri Lanka, and earlier reports of this species from India probably refers to a species of the $C$. gachua complex. For details, see http://fl.biology.usgs.gov/Snakehead_circ_1251/html/channa_orientalis. html

INT Refers to introduced species, and hence IUCN status is not provided. IUCN status: LC - Least Concern; NT - Near Threatened; VU - Vulnerable; EN Endangered; CR - Critically Endangered.

References: A - Dubey \& Mehra (1959); B - Molur \& Walker (1998a); C - Saksena (2007); D - Sale (1982); E - Sharma \& Choudhury (2007); F - Sivakumar (2002); G - Srivastava (2007); H - Vyas \& Singh (2004); I - This study (2009-2010); J - N Kelkar pers. comm.; K - Sivakumar \& Choudhury (2008). river, due to which species become more vulnerable to netting and dynamiting (Dubey \& Mehra 1959; Katdare et al. 2011). Additionally, reduction in the number of inaccessible islands results in increased destruction of nests of Gharials, turtles and ground-nesting birds like skimmers and Black-bellied Terns (Sundar 2004; Nair 2010). Altered flow regimes, and insufficient flooding disrupts siltation rates and sand deposition in the river channel. As Moll (1997) notes, upriver dams exacerbate the problem by preventing replacement sand from coming downriver while increasing erosion by periodic and unseasonable elevation of water levels.

Sand-mining destroys crucial breeding areas and is one of the most serious threats to the survival of species that lay their eggs on sand deposits. Stonemining, common in the upper sections of the river, causes considerable disturbances to wildlife, destroys key breeding habitats like otter-holts and provides easy access to ammunition for dynamite fishing (Katdare et al. 2011).

Poaching is another issue that continues unchecked (Murthy 2004; Tarun Nair 2009-2013 pers. obs.) due to inadequate allocation of field personnel to patrol the sanctuary. Illegal fishing and turtle poaching are rampant, using a variety of methods (gill net, baited hook-line, dynamite) and these also claim other species like Gharials, Mugger, river dolphins, otters and several birds (Dubey \& Mehra 1959; Vyas 2004; Nair 2010; Taigor \& Rao 2010; Katdare et al. 2011). Gill nets are particularly responsible for entangling and drowning juvenile Gharials, thereby impacting survival and recruitment of smaller size-classes.

Riparian agriculture and associated activities like constant human disturbance from irrigational pump operation and crop protection, and risks of water pollution from agro-chemical use and oil leaks also contribute substantially to habitat loss, degradation and pollution (Katdare et al. 2011).

In the future, river flows would be further impacted by the 52 irrigation projects that are under construction and 376 projects that have been planned in the basin (Department of Water Resources, Rajasthan). Additionally, there are proposals to divert the two most important tributaries of the Chambal - the Parbati and Kalisindh rivers (NWDA). Inspite of water being the most critical resource in the NCS, the environmental impact assessment for this project does not account for changes in the hydrological regime due to the diversion of water (NWDA). There have also been calls to denotify the sanctuary itself in order to facilitate sand-mining (The 
Table 3. Checklist of reptiles of the Chambal River Basin

\begin{tabular}{|c|c|c|c|c|}
\hline & Species & Common Name & $\begin{array}{l}\text { IUCN status } \\
2009-2012\end{array}$ & References \\
\hline & Crocodylidae & & & \\
\hline \multirow[t]{2}{*}{1} & Crocodylus palustris (Lesson, 1831) & Mugger or Marsh Crocodile & VU & $\mathrm{A}, \mathrm{G}, \mathrm{H}, \mathrm{M}$ \\
\hline & Gavialidae & & & \\
\hline \multirow[t]{2}{*}{2} & Gavialis gangeticus (Gmelin, 1789) & Gharial & CR & $A, G, H, M$ \\
\hline & Geoemydidae & & & \\
\hline 3 & Batagur dhongoka (Gray, 1832) & Three-striped Roofed Turtle & EN & $A, G, M$ \\
\hline 4 & Batagur kachuga (Gray, 1831) & Red-crowned Roofed Turtle & CR & $A, G, M$ \\
\hline 5 & Hardella thurjii (Gray, 1831) & Crowned River Turtle & VU & $A, G, M$ \\
\hline 6 & Pangshura tecta (Gray, 1830) & Indian Roofed Turtle & LC & $\mathrm{K}, \mathrm{M}$ \\
\hline \multirow[t]{2}{*}{7} & Pangshura tentoria (Gray, 1834) & Indian Tent Turtle & LC & $A, G, M$ \\
\hline & Testudinidae & & & \\
\hline \multirow[t]{2}{*}{8} & Geochelone elegans (Schoepff, 1795) & Indian Star Tortoise & LC & $\mathrm{H}, \mathrm{L}$ \\
\hline & Trionychidae & & & \\
\hline 9 & Lissemys punctata (Bonnaterre, 1789) & Indian Flapshell Turtle & LC & $\mathrm{A}, \mathrm{G}, \mathrm{H}, \mathrm{M}$ \\
\hline 10 & Chitra indica (Gray, 1830) & Narrow-headed Softshell Turtle & EN & $A, G, M$ \\
\hline 11 & Nilssonia gangetica (Cuvier, 1825) & Indian Softshell Turtle & VU & $A, G, H, M$ \\
\hline \multirow[t]{2}{*}{12} & Nilssonia hurum (Gray, 1830) & Peacock Softshell Turtle & VU & C \\
\hline & Gekkonidae & & & \\
\hline 13 & Hemidactylus brookii (Gray, 1845) & Brook's House Gecko & - & A \\
\hline 14 & Hemidactylus flaviviridis Rüppell, 1840 & Yellow-bellied House Gecko & - & $\mathrm{A}, \mathrm{H}, \mathrm{M}$ \\
\hline 15 & Hemidactylus frenatus Duméril \& Bibron, 1836 & Common House Gecko & LC & A \\
\hline 16 & Hemidactylus maculatus Duméril \& Bibron, 1836 & Northern Spotted Gecko & - & M \\
\hline 17 & Hemidactylus leschenaultii Duméril \& Bibron, 1836 & Bark Gecko & - & $A, M$ \\
\hline \multirow[t]{2}{*}{18} & Hemidactylus triedrus (Daudin, 1802) & Termite Hill Gecko & - & A \\
\hline & Agamidae & & & \\
\hline 19 & Calotes versicolor (Daudin, 1802) & Oriental Garden Lizard & - & $\mathrm{A}, \mathrm{H}, \mathrm{M}$ \\
\hline \multirow[t]{2}{*}{20} & Sitana ponticeriana Cuvier, 1829 & Fan-throated Lizard & & A \\
\hline & Chamaeleonidae & & & \\
\hline \multirow[t]{2}{*}{21} & Chamaeleo zeylanicus Laurenti, 1768 & Indian Chameleon & - & A \\
\hline & Varanidae & & & \\
\hline 22 & Varanus bengalensis (Daudin, 1802) & Common Indian Monitor & LC & $A, F, H, L, M$ \\
\hline \multirow[t]{2}{*}{23} & Varanus griseus Mertens, 1954 & Indian Desert Monitor & LC & $\mathrm{H}$ \\
\hline & Lacertidae & & & \\
\hline 24 & Ophisops jerdonii Blyth, 1853 & Jerdon's Snake-eye & LC & $A, D$ \\
\hline 25 & Ophisops microlepis Blanford, 1870 & Small-scaled Lacerta & LC & B \\
\hline \multirow[t]{2}{*}{26} & Ophisops minor nictans Arnold, 1989 & Indian Dwarf Lacerta & - & $A, D$ \\
\hline & Scincidae & & & \\
\hline 27 & Eutropis carinata (Schneider, 1801) & Keeled Grass Skink & - & $A, M$ \\
\hline 28 & Eutropis innotata (Blanford, 1870) & Blanford's Grass Skink & - & D \\
\hline
\end{tabular}




\begin{tabular}{|c|c|c|c|c|}
\hline & Species & Common Name & $\begin{array}{l}\text { IUCN status } \\
2009-2012\end{array}$ & References \\
\hline 29 & Eutropis macularia (Blyth, 1853) & Bronze Skink & - & A \\
\hline \multirow[t]{2}{*}{30} & Lygosoma punctata (Gmelin, 1799) & Spotted Supple Skink & - & $A, M$ \\
\hline & Typhlopidae & & & \\
\hline \multirow[t]{2}{*}{31} & Ramphotyphlops braminus (Daudin, 1803) & Brahminy Blind snake & - & A \\
\hline & Pythonidae & & & \\
\hline \multirow[t]{2}{*}{32} & Python molurus (Linnaeus, 1758) & Indian Rock Python & NT & $\mathrm{K}, \mathrm{M}$ \\
\hline & Boidae & & & \\
\hline 33 & Eryx johnii (Russell, 1801) & Red Sand Boa & - & $A, M$ \\
\hline \multirow[t]{2}{*}{34} & Gongylophis conicus (Schneider, 1801) & Common Sand Boa & - & M \\
\hline & Colubridae & & & \\
\hline 35 & Coelognathus helena (Daudin, 1803) & Common Trinket Snake & - & $\mathrm{N}$ \\
\hline 36 & Ptyas mucosa (Linnaeus, 1758) & Indian Rat Snake & - & $\mathrm{A}, \mathrm{H}, \mathrm{M}$ \\
\hline 37 & Platyceps ventromaculatus (Gray, 1834) & Glossy-bellied Racer & - & I \\
\hline 38 & Argyrogena fasciolata (Shaw, 1802) & Banded Racer & - & $\mathrm{N}$ \\
\hline 39 & Spalerosophis atriceps Fischer, 1885 & Black-headed Royal Snake & - & $\mathrm{N}$ \\
\hline 40 & Oligodon taeniolatus (Jerdon, 1853) & Russell's Kukri Snake & LC & A \\
\hline 41 & Oligodon arnensis (Shaw, 1802) & Common Kukri Snake & - & $\mathrm{A}, \mathrm{N}$ \\
\hline 42 & Dendrelaphis tristis (Daudin, 1803) & Common Bronzeback Tree Snake & - & $\mathrm{N}$ \\
\hline 43 & Lycodon striatus (Shaw, 1802) & Barred Wolf Snake & - & $\mathrm{N}$ \\
\hline 44 & Lycodon aulicus (Linnaeus, 1758) & Common Wolf Snake & - & $A, N$ \\
\hline 45 & Sibynophis subpunctatus (Dumeril Bibron \& Dumeril, 1854) & Dumeril's Black-headed Snake & - & J \\
\hline 46 & Xenochrophis piscator (Schneider, 1799) & Chequered Keelback & - & $\mathrm{A}, \mathrm{M}, \mathrm{N}$ \\
\hline 47 & Amphiesma stolata (Linnaeus, 1758) & Buff Striped Keelback & - & $\mathrm{A}, \mathrm{N}$ \\
\hline 48 & Macropisthodon plumbicolor (Cantor, 1839) & Green Keelback & - & $\mathrm{N}$ \\
\hline 49 & Boiga trigonata (Schneider, 1802) & Common Cat Snake & LC & $\mathrm{N}$ \\
\hline \multirow[t]{2}{*}{50} & Ahaetulla nasuta (Bonnaterre, 1790) & Common Vine Snake & & $\mathrm{A}, \mathrm{N}$ \\
\hline & Elapidae & & & \\
\hline 51 & Bungarus caeruleus (Schneider, 1801) & Common Krait & - & $\mathrm{A}, \mathrm{H}, \mathrm{N}$ \\
\hline \multirow[t]{2}{*}{52} & Naja naja (Linnaeus, 1758) & Spectacled Cobra & - & $\mathrm{A}, \mathrm{N}$ \\
\hline & Homalopsidae & & & \\
\hline \multirow[t]{2}{*}{53} & Enhydris sieboldii (Schlegel, 1837) & Siebold's Smooth-water Snake & LC & $\mathrm{E}$ \\
\hline & Psammophiidae & & & \\
\hline \multirow[t]{2}{*}{54} & Psammophis leithii Gunther, 1869 & Leith's Sand Snake & - & $\mathrm{N}$ \\
\hline & Viperidae & & & \\
\hline 55 & Daboia russelii (Shaw \& Nodder, 1797) & Russell's Viper & - & $F, M$ \\
\hline 56 & Echis carinatus (Schneider, 1801) & Saw-scaled Viper & - & $A, N$ \\
\hline
\end{tabular}

IUCN status: LC - Least Concern; NT - Near Threatened; VU - Vulnerable; EN - Endangered; CR - Critically Endangered.

References: A - This study (fieldwork in 2009 - 2010); B - Böhm \& Richman (2010); C - Das et al. (2010); D - Molur \& Walker (1998b); E - Murphy \& Lobo (2010); F Nair (2009); G - Rao (1988); H - Sale (1982); I - Schätti \& Schmitz (2006); J - Sharma (2003); K - Vyas (2004); L - Vyas \& Singh (2004); M - Vyas et al. (in prep.); N - This study (intermittent fieldwork between 2006 and 2008). 
Table 4. Checklist of birds of the Chambal River Basin

\begin{tabular}{|c|c|c|c|c|}
\hline & Species & Common Name & $\begin{array}{c}\text { IUCN Status } \\
2011\end{array}$ & References \\
\hline & Phasianidae & & & \\
\hline 1 & Francolinus francolinus (Linnaeus, 1766) & Black Francolin & LC & $\mathrm{H}$ \\
\hline 2 & Francolinus pondicerianus (Gmelin, 1789) & Grey Francolin & LC & $\mathrm{A}, \mathrm{B}, \mathrm{G}, \mathrm{H}, \mathrm{K}, \mathrm{L}, \mathrm{M}$ \\
\hline 3 & Coturnix coturnix (Linnaeus, 1758) & Common Quail & LC & $\mathrm{A}, \mathrm{B}, \mathrm{H}, \mathrm{M}$ \\
\hline 4 & Coturnix coromandelica (Gmelin, 1789) & Rain Quail & LC & $\mathrm{H}$ \\
\hline 5 & Perdicula asiatica (Latham, 1790) & Jungle Bush-quail & LC & $\mathrm{H}$ \\
\hline 6 & Galloperdix spadicea (Gmelin, 1789) & Red Spurfowl & LC & B \\
\hline 7 & Galloperdix lunulata (Valenciennes, 1825) & Painted Spurfowl & LC & $\mathrm{A}, \mathrm{M}$ \\
\hline 8 & Gallus sonneratii Temminck, 1813 & Grey Junglefowl & LC & $A, B$ \\
\hline \multirow[t]{2}{*}{9} & Pavo cristatus Linnaeus, 1758 & Indian Peafowl & LC & $\mathrm{A}, \mathrm{B}, \mathrm{G}, \mathrm{H}, \mathrm{K}, \mathrm{L}, \mathrm{M}$ \\
\hline & Anatidae & & & \\
\hline 10 & Dendrocygna javanica (Horsfield, 1821) & Lesser Whistling-duck & LC & $\mathrm{A}, \mathrm{B}, \mathrm{G}, \mathrm{I}, \mathrm{K}, \mathrm{M}$ \\
\hline 11 & Anser anser (Linnaeus, 1758) & Greylag Goose & LC & $\mathrm{A}, \mathrm{H}, \mathrm{I}, \mathrm{L}, \mathrm{M}$ \\
\hline 12 & Anser indicus (Latham, 1790) & Bar-headed Goose & LC & $A, B, H, I, K, L, M$ \\
\hline 13 & Sarkidiornis melanotos (Pennant, 1769) & Comb Duck & LC & $\mathrm{A}, \mathrm{B}, \mathrm{G}, \mathrm{H}, \mathrm{I}, \mathrm{K}, \mathrm{M}$ \\
\hline 14 & Tadorna ferruginea (Pallas, 1764) & Ruddy Shelduck & LC & $A, B, G, H, K, L, M$ \\
\hline 15 & Tadorna tadorna (Linnaeus, 1758) & Common Shelduck & LC & K \\
\hline 16 & Nettapus coromandelianus (Gmelin, 1789) & Cotton Pygmy-goose & LC & A \\
\hline 17 & Anas strepera Linnaeus, 1758 & Gadwall & LC & $\mathrm{K}, \mathrm{L}, \mathrm{M}$ \\
\hline 18 & Anas penelope Linnaeus, 1758 & Eurasian Wigeon & LC & $L, M$ \\
\hline 19 & Anas platyrhynchos Linnaeus, 1758 & Mallard & LC & $A, H$ \\
\hline 20 & Anas poecilorhyncha Forster, 1781 & Western Spot-billed Duck & LC & $\mathrm{A}, \mathrm{B}, \mathrm{G}, \mathrm{H}, \mathrm{K}, \mathrm{L}, \mathrm{M}$ \\
\hline 21 & Anas clypeata Linnaeus, 1758 & Northern Shoveler & LC & $A, B, I, L, M$ \\
\hline 22 & Anas acuta Linnaeus, 1758 & Northern Pintail & LC & $\mathrm{A}, \mathrm{I}, \mathrm{K}, \mathrm{L}, \mathrm{M}$ \\
\hline 23 & Anas querquedula Linnaeus, 1758 & Garganey & LC & $A, B, G, L, M$ \\
\hline 24 & Anas crecca Linnaeus, 1758 & Common Teal & LC & $\mathrm{A}, \mathrm{H}, \mathrm{I}, \mathrm{K}, \mathrm{L}, \mathrm{M}$ \\
\hline 25 & Mergus merganser Linnaeus, 1758 & Common Merganser & LC & M \\
\hline 26 & Netta rufina (Pallas, 1773) & Red-crested Pochard & LC & $\mathrm{A}, \mathrm{B}, \mathrm{H}, \mathrm{I}, \mathrm{K}, \mathrm{M}$ \\
\hline 27 & Aythya ferina (Linnaeus, 1758) & Common Pochard & LC & $L, M$ \\
\hline 28 & Aythya nyroca (Gldenstdt, 1770) & Ferruginous Duck & NT & $A, H, K$ \\
\hline \multirow[t]{2}{*}{29} & Aythya fuligula (Linnaeus, 1758) & Tufted Duck & LC & $\mathrm{A}, \mathrm{H}, \mathrm{L}$ \\
\hline & Podicipedidae & & & \\
\hline 30 & Tachybaptus ruficollis (Pallas, 1764) & Little Grebe & LC & $\mathrm{A}, \mathrm{B}, \mathrm{G}, \mathrm{H}, \mathrm{K}, \mathrm{L}, \mathrm{M}$ \\
\hline \multirow[t]{2}{*}{31} & Podiceps cristatus (Linnaeus, 1758) & Great Crested Grebe & LC & $A, B, K$ \\
\hline & Phoenicopteridae & & & \\
\hline \multirow[t]{2}{*}{32} & Phoenicopterus roseus Pallas, 1811 & Greater Flamingo & LC & $\mathrm{A}, \mathrm{M}$ \\
\hline & Ciconiidae & & & \\
\hline 33 & Mycteria leucocephala (Pennant, 1769) & Painted Stork & NT & $A, B, G, H, K, L, M$ \\
\hline 34 & Anastomus oscitans (Boddaert, 1783) & Asian Openbill & LC & $\mathrm{A}, \mathrm{B}, \mathrm{G}, \mathrm{H}, \mathrm{I}, \mathrm{K}, \mathrm{M}$ \\
\hline 35 & Ciconia nigra (Linnaeus, 1758) & Black stork & LC & K,L,M \\
\hline 36 & Ciconia episcopus (Boddaert, 1783) & Woolly-necked Stork & LC & $A, B, G, H, K, L, M$ \\
\hline 37 & Ephippiorhynchus asiaticus (Latham, 1790) & Black-necked Stork & NT & $A, B, H, I$ \\
\hline \multirow[t]{2}{*}{38} & Leptoptilos javanicus (Horsfield, 1821) & Lesser Adjutant & VU & E \\
\hline & Threskiornithidae & & & \\
\hline 39 & Threskiornis melanocephalus (Latham, 1790) & Black-headed Ibis & NT & $\mathrm{A}, \mathrm{B}, \mathrm{G}, \mathrm{I}, \mathrm{K}, \mathrm{M}$ \\
\hline 40 & Pseudibis papillosa (Temminck, 1824) & Red-naped Ibis & LC & $\mathrm{A}, \mathrm{B}, \mathrm{G}, \mathrm{I}, \mathrm{K}, \mathrm{L}, \mathrm{M}$ \\
\hline
\end{tabular}




\begin{tabular}{|c|c|c|c|c|}
\hline & Species & Common Name & $\begin{array}{c}\text { IUCN Status } \\
2011\end{array}$ & References \\
\hline 41 & Plegadis falcinellus (Linnaeus, 1766) & Glossy Ibis & LC & $\mathrm{A}, \mathrm{G}, \mathrm{K}, \mathrm{M}$ \\
\hline \multirow[t]{2}{*}{42} & Platalea leucorodia Linnaeus, 1758 & Eurasian Spoonbill & LC & $A, B, G, I, K, L, M$ \\
\hline & Ardeidae & & & \\
\hline 43 & Botaurus stellaris (Linnaeus, 1758) & Great Bittern & LC & A \\
\hline 44 & Ixobrychus sinensis (Gmelin, 1789) & Yellow Bittern & LC & B \\
\hline 45 & Ixobrychus cinnamomeus (Gmelin, 1789) & Cinnamon Bittern & LC & M \\
\hline 46 & Ixobrychus flavicollis (Latham, 1790) & Black Bittern & LC & A \\
\hline 47 & Nycticorax nycticorax (Linnaeus, 1758) & Black-crowned Night-heron & LC & $A, B, G, K, M$ \\
\hline 48 & Butorides striata (Linnaeus, 1758) & Little Heron & LC & $A, B, K, L, M$ \\
\hline 49 & Ardeola grayii (Sykes, 1832) & Indian Pond-heron & LC & $A, B, G, H, K, L, M$ \\
\hline 50 & Bubulcus ibis (Linnaeus, 1758) & Cattle Egret & LC & $\mathrm{A}, \mathrm{B}, \mathrm{G}, \mathrm{H}, \mathrm{K}, \mathrm{L}, \mathrm{M}$ \\
\hline 51 & Ardea cinerea Linnaeus, 1758 & Grey Heron & LC & $\mathrm{A}, \mathrm{B}, \mathrm{G}, \mathrm{H}, \mathrm{K}, \mathrm{L}, \mathrm{M}$ \\
\hline 52 & Ardea purpurea Linnaeus, 1766 & Purple Heron & LC & $A, B, G, K, M$ \\
\hline 53 & Casmerodius albus (Linnaeus, 1758) & Great Egret & LC & $B, G, K, L, M$ \\
\hline 54 & Mesophoyx intermedia (Wagler, 1829) & Intermediate Egret & LC & $A, G, K, L, M$ \\
\hline \multirow[t]{2}{*}{55} & Egretta garzetta (Linnaeus, 1766) & Little Egret & LC & $\mathrm{A}, \mathrm{B}, \mathrm{G}, \mathrm{H}, \mathrm{K}, \mathrm{L}, \mathrm{M}$ \\
\hline & Pelecanidae & & & \\
\hline 56 & Pelecanus onocrotalus Linnaeus, 1758 & Great White Pelican & LC & K \\
\hline 57 & Pelecanus crispus Bruch, 1832 & Dalmatian Pelican & VU & $A, B, E$ \\
\hline \multirow[t]{2}{*}{58} & Pelecanus philippensis Gmelin, 1789 & Spot-billed Pelican & NT & $A, E, H, I$ \\
\hline & Phalacrocoridae & & & \\
\hline 59 & Phalacrocorax niger (Vieillot, 1817) & Little Cormorant & LC & $\mathrm{A}, \mathrm{B}, \mathrm{G}, \mathrm{H}, \mathrm{K}, \mathrm{L}, \mathrm{M}$ \\
\hline 60 & Phalacrocorax fuscicollis Stephens, 1826 & Indian Cormorant & LC & $A, B, G, L, M$ \\
\hline \multirow[t]{2}{*}{61} & Phalacrocorax carbo (Linnaeus, 1758) & Great Cormorant & LC & $A, B, G, I, K, L, M$ \\
\hline & Anhingidae & & & \\
\hline \multirow[t]{2}{*}{62} & Anhinga melanogaster Pennant, 1769 & Oriental Darter & NT & $\mathrm{A}, \mathrm{B}, \mathrm{G}, \mathrm{H}, \mathrm{K}, \mathrm{L}, \mathrm{M}$ \\
\hline & Falconidae & & & \\
\hline 63 & Falco naumanni Fleischer, 1818 & Lesser Kestrel & LC & $\mathrm{E}$ \\
\hline 64 & Falco tinnunculus Linnaeus, 1758 & Common Kestrel & LC & $A, B, H, L, M$ \\
\hline 65 & Falco chicquera Daudin, 1800 & Red-necked Falcon & LC & A \\
\hline 66 & Falco subbuteo Linnaeus, 1758 & Eurasian Hobby & LC & M \\
\hline 67 & Falco jugger Gray, 1834 & Laggar Falcon & NT & $\mathrm{H}, \mathrm{M}$ \\
\hline \multirow[t]{2}{*}{68} & Falco peregrinus Tunstall, 1771 & Peregrine Falcon & LC & $A, B, M$ \\
\hline & Accipitridae & & & \\
\hline 69 & Pandion haliaetus (Linnaeus, 1758) & Osprey & LC & $\mathrm{A}, \mathrm{B}, \mathrm{G}, \mathrm{H}, \mathrm{K}, \mathrm{L}, \mathrm{M}$ \\
\hline 70 & Pernis ptilorhyncus (Temminck, 1821) & Oriental Honey-buzzard & LC & $\mathrm{B}, \mathrm{K}, \mathrm{M}$ \\
\hline 71 & Elanus caeruleus (Desfontaines, 1789) & Black-winged Kite & LC & $\mathrm{A}, \mathrm{B}, \mathrm{G}, \mathrm{H}, \mathrm{K}, \mathrm{L}, \mathrm{M}$ \\
\hline 72 & Milvus migrans (Boddaert, 1783) & Black Kite & LC & $\mathrm{B}, \mathrm{G}, \mathrm{L}, \mathrm{M}$ \\
\hline 73 & Haliastur indus (Boddaert, 1783) & Brahminy Kite & LC & B \\
\hline 74 & Haliaeetus leucoryphus (Pallas, 1771) & Pallas's Fish-eagle & VU & $C, D, F$ \\
\hline 75 & Haliaeetus albicilla (Linnaeus, 1758) & White-tailed Eagle & LC & B \\
\hline 76 & Neophron percnopterus (Linnaeus, 1758) & Egyptian Vulture & EN & $\mathrm{A}, \mathrm{B}, \mathrm{G}, \mathrm{H}, \mathrm{K}, \mathrm{L}, \mathrm{M}$ \\
\hline 77 & Gyps bengalensis (Gmelin, 1788) & White-rumped Vulture & CR & $\mathrm{B}, \mathrm{D}, \mathrm{F}, \mathrm{G}, \mathrm{H}, \mathrm{L}, \mathrm{M}$ \\
\hline 78 & Gyps indicus (Scopoli, 1786) & Indian Vulture & CR & $\mathrm{B}, \mathrm{D}, \mathrm{F}, \mathrm{G}, \mathrm{K}, \mathrm{L}, \mathrm{M}$ \\
\hline 79 & Gyps tenuirostris Gray, 1844 & Slender-billed Vulture & CR & L \\
\hline 80 & Gyps fulvus (Hablizl, 1783) & Griffon Vulture & LC & $\mathrm{G}, \mathrm{L}, \mathrm{M}$ \\
\hline 81 & Sarcogyps calvus (Scopoli, 1786) & Red-headed Vulture & CR & $A, G, I, K, L, M$ \\
\hline
\end{tabular}




\begin{tabular}{|c|c|c|c|c|}
\hline & Species & Common Name & $\begin{array}{l}\text { IUCN Status } \\
2011\end{array}$ & References \\
\hline 82 & Circaetus gallicus (Gmelin, 1788) & Short-toed snake eagle & LC & $\mathrm{A}, \mathrm{H}, \mathrm{K}, \mathrm{M}$ \\
\hline 83 & Spilornis cheela (Latham, 1790) & Crested Serpent-eagle & LC & $B, K, L, M$ \\
\hline 84 & Circus aeruginosus (Linnaeus, 1758) & Western Marsh-harrier & LC & $A, B, G, H, K, L, M$ \\
\hline 85 & Circus macrourus (Gmelin, 1770) & Pallid Harrier & NT & $\mathrm{H}, \mathrm{K}, \mathrm{M}$ \\
\hline 86 & Circus pygargus (Linnaeus, 1758) & Montagu's Harrier & LC & $\mathrm{L}$ \\
\hline 87 & Accipiter badius (Gmelin, 1788) & Shikra & LC & $A, B, G, K, L, M$ \\
\hline 88 & Accipiter nisus (Linnaeus, 1758) & Eurasian Sparrowhawk & $\mathrm{LC}$ & $\mathrm{k}$ \\
\hline 89 & Butastur teesa (Franklin, 1831) & White-eyed Buzzard & LC & $H, L, M$ \\
\hline 90 & Aquila pomarina Brehm, 1831 & Lesser Spotted Eagle & LC & A \\
\hline 91 & Aquila clanga Pallas, 1811 & Greater Spotted Eagle & Vu & $A, C, D, E, F, M$ \\
\hline 92 & Aquila rapax (Temminck, 1828) & Tawny Eagle & LC & $\mathrm{B}, \mathrm{H}$ \\
\hline 93 & Aquila nipalensis Hodgson, 1833 & Steppe Eagle & LC & B \\
\hline 94 & Aquila heliaca Savigny, 1809 & Eastern Imperial Eagle & vu & $E, L, M$ \\
\hline 95 & Aquila fasciatus (Vieillot, 1822) & Bonelli's Eagle & LC & M \\
\hline 96 & Hieraaetus pennatus (Gmelin, 1788) & Booted Eagle & LC & $A, B$ \\
\hline \multirow[t]{2}{*}{97} & Nisaetus cirrhatus (Gmelin, 1788) & Changeable Hawk-eagle & $\mathrm{LC}$ & $A, B, H$ \\
\hline & Rallidae & & & \\
\hline 98 & Amaurornis akool (Sykes, 1832) & Brown Crake & LC & $\mathrm{A}, \mathrm{K}, \mathrm{M}$ \\
\hline 99 & Amaurornis phoenicurus (Pennant, 1769) & White-breasted Waterhen & $\mathrm{LC}$ & $A, B, L, M$ \\
\hline 100 & Porzana pusilla (Pallas, 1776) & Baillon's Crake & LC & A \\
\hline 101 & Porphyrio porphyrio (Linnaeus, 1758) & Purple Swamphen & LC & $\mathrm{A}, \mathrm{B}, \mathrm{G}, \mathrm{M}$ \\
\hline 102 & Gallinula chloropus (Linnaeus, 1758) & Common Moorhen & LC & M \\
\hline \multirow[t]{2}{*}{103} & Fulica atra Linnaeus, 1758 & Common Coot & LC & $A, B, G, K, L, M$ \\
\hline & Gruidae & & & \\
\hline 104 & Grus antigone (Linnaeus, 1758) & Sarus Crane & vu & $A, B, C, D, E, F, G, H, I, L, M$ \\
\hline 105 & Grus virgo (Linnaeus, 1758) & Demoiselle Crane & $\mathrm{LC}$ & M \\
\hline \multirow[t]{2}{*}{106} & Grus grus (Linnaeus, 1758) & Common Crane & LC & $\mathrm{I}, \mathrm{L}$ \\
\hline & Turnicidae & & & \\
\hline 107 & Turnix sylvaticus (Desfontaines, 1787) & Small Buttonquail & LC & A \\
\hline 108 & Turnix tanki Blyth, 1843 & Yellow-legged Buttonquail & LC & L,M \\
\hline \multirow[t]{2}{*}{109} & Turnix suscitator (Gmelin, 1789) & Barred Buttonquail & LC & $\mathrm{H}$ \\
\hline & Burhinidae & & & \\
\hline 110 & Esacus recurvirostris (Cuvier, 1829) & Great Thick-knee & $\mathrm{LC}$ & $\mathrm{A}, \mathrm{B}, \mathrm{I}, \mathrm{K}, \mathrm{L}, \mathrm{M}$ \\
\hline \multirow[t]{2}{*}{111} & Burhinus oedicnemus (Linnaeus, 1758) & Eurasian Thick-knee & LC & $\mathrm{A}, \mathrm{B}, \mathrm{H}, \mathrm{M}$ \\
\hline & Recurvirostridae & & & \\
\hline 112 & Himantopus himantopus (Linnaeus, 1758) & Black-winged Stilt & LC & $\mathrm{A}, \mathrm{B}, \mathrm{G}, \mathrm{H}, \mathrm{K}, \mathrm{L}, \mathrm{M}$ \\
\hline \multirow[t]{2}{*}{113} & Recurvirostra avosetta Linnaeus, 1758 & Pied Avocet & $\mathrm{LC}$ & $\begin{array}{c}\text { (Rakesh Vyas, pers. } \\
\text { comm.) }\end{array}$ \\
\hline & Charadriidae & & & \\
\hline 114 & Vanellus malarbaricus (Boddaert, 1783) & Yellow-wattled Lapwing & LC & $A, B, G$ \\
\hline 115 & Vanellus duvaucelii (Lesson, 1826) & River Lapwing & LC & $\mathrm{A}, \mathrm{B}, \mathrm{G}, \mathrm{K}, \mathrm{M}$ \\
\hline 116 & Vanellus indicus (Boddaert, 1783) & Red-wattled Lapwing & $\mathrm{LC}$ & $\mathrm{A}, \mathrm{B}, \mathrm{G}, \mathrm{H}, \mathrm{K}, \mathrm{L}, \mathrm{M}$ \\
\hline 117 & Vanellus leucurus (Lichtenstein, 1823) & White-tailed Lapwing & LC & $A, G, L$ \\
\hline 118 & Charadrius dubius Scopoli, 1786 & Little Ringed Plover & LC & $\mathrm{A}, \mathrm{B}, \mathrm{G}, \mathrm{H}, \mathrm{K}, \mathrm{L}, \mathrm{M}$ \\
\hline \multirow[t]{2}{*}{119} & Charadrius alexandrinus Linnaeus, 1758 & Kentish Plover & LC & $A, K, L, M$ \\
\hline & Rostratulidae & & & \\
\hline \multirow[t]{2}{*}{120} & Rostratula benghalensis (Linnaeus, 1758) & Greater Painted-snipe & LC & $\mathrm{A}, \mathrm{M}$ \\
\hline & Jacanidae & & & \\
\hline 121 & Hydrophasianus chirurgus (Scopoli, 1786) & Pheasant-tailed Jacana & LC & $\mathrm{A}, \mathrm{K}, \mathrm{M}$ \\
\hline
\end{tabular}




\begin{tabular}{|c|c|c|c|c|}
\hline & Species & Common Name & $\begin{array}{c}\text { IUCN Status } \\
2011\end{array}$ & References \\
\hline \multirow[t]{2}{*}{122} & Metopidius indicus (Latham, 1790) & Bronze-winged Jacana & LC & M \\
\hline & Scolopacidae & & & \\
\hline 123 & Gallinago gallinago (Linnaeus, 1758) & Common Snipe & LC & L,M \\
\hline 124 & Limosa limosa (Linnaeus, 1758) & Black-tailed Godwit & NT & $A, G, K, L, M$ \\
\hline 125 & Numenius arquata (Linnaeus, 1758) & Eurasian Curlew & NT & $\mathrm{K}, \mathrm{L}, \mathrm{M}$ \\
\hline 126 & Tringa erythropus (Pallas, 1764) & Spotted Redshank & LC & L \\
\hline 127 & Tringa totanus (Linnaeus, 1758) & Common Redshank & LC & $\mathrm{A}, \mathrm{G}, \mathrm{H}, \mathrm{K}, \mathrm{L}, \mathrm{M}$ \\
\hline 128 & Tringa stagnatilis (Bechstein, 1803) & Marsh Sandpiper & LC & L \\
\hline 129 & Tringa nebularia (Gunnerus, 1767) & Common Greenshank & LC & $A, G, K, L, M$ \\
\hline 130 & Tringa ochropus Linnaeus, 1758 & Green Sandpiper & LC & A,K,L,M \\
\hline 131 & Tringa glareola Linnaeus, 1758 & Wood Sandpiper & LC & $A, B, G, K, L, M$ \\
\hline 132 & Actitis hypoleucos Linnaeus, 1758 & Common Sandpiper & LC & $\mathrm{A}, \mathrm{B}, \mathrm{G}, \mathrm{H}, \mathrm{K}, \mathrm{L}, \mathrm{M}$ \\
\hline 133 & Calidris minuta (Leisler, 1812) & Little Stint & LC & $\mathrm{A}, \mathrm{G}, \mathrm{H}, \mathrm{K}, \mathrm{L}, \mathrm{M}$ \\
\hline 134 & Calidris temminckii (Leisler, 1812) & Temminck's Stint & LC & $L, M$ \\
\hline \multirow[t]{2}{*}{135} & Philomachus pugnax (Linnaeus, 1758) & Ruff & LC & $\mathrm{A}, \mathrm{G}, \mathrm{H}, \mathrm{L}, \mathrm{M}$ \\
\hline & Glareolidae & & & \\
\hline 136 & Cursorius coromandelicus (Gmelin, 1789) & Indian Courser & LC & A \\
\hline 137 & Glareola pratincola (Linnaeus, 1766) & Collared Pratincole & LC & $A, M$ \\
\hline \multirow[t]{2}{*}{138} & Glareola lactea Temminck, 1820 & Small Pratincole & LC & $\mathrm{A}, \mathrm{G}, \mathrm{H}, \mathrm{J}, \mathrm{K}, \mathrm{M}$ \\
\hline & Laridae & & & \\
\hline 139 & Larus cachinnans Pallas, 1811 & Caspian Gull & LC & $\mathrm{K}, \mathrm{M}$ \\
\hline 140 & Larus ichthyaetus Pallas, 1773 & Pallas's Gull & LC & A,K,L,M \\
\hline 141 & Larus brunnicephalus Jerdon, 1840 & Brown-headed Gull & LC & $A, H, I, K, L, M$ \\
\hline 142 & Larus ridibundus Linnaeus, 1766 & Black-headed Gull & LC & $\mathrm{K}, \mathrm{L}, \mathrm{M}$ \\
\hline 143 & Sterna aurantia Gray, 1831 & River Tern & LC & $\mathrm{A}, \mathrm{B}, \mathrm{G}, \mathrm{H}, \mathrm{K}, \mathrm{L}, \mathrm{M}$ \\
\hline 144 & Sterna albifrons Pallas, 1764 & Little Tern & LC & $A, G, J$ \\
\hline 145 & Sterna acuticauda Gray, 1832 & Black-bellied Tern & NT & $A, G, K, L, M$ \\
\hline 146 & Chlidonias hybrida (Pallas, 1811) & Whiskered Tern & LC & $\mathrm{A}, \mathrm{G}, \mathrm{H}, \mathrm{K}, \mathrm{L}, \mathrm{M}$ \\
\hline \multirow[t]{2}{*}{147} & Rynchops albicollis Swainson, 1838 & Indian Skimmer & VU & A,B.C.D,E,F,G,I,J,K,M \\
\hline & Pteroclididae & & & \\
\hline 148 & Pterocles exustus Temminck, 1825 & Chestnut-bellied Sandgrouse & LC & $A, G, H, M$ \\
\hline \multirow[t]{2}{*}{149} & Pterocles indicus (Gmelin, 1789) & Painted Sandgrouse & LC & K \\
\hline & Columbidae & & & \\
\hline 150 & Columba livia Gmelin, 1789 & Rock Pigeon & LC & $A, B, G, H, K, L, M$ \\
\hline 151 & Streptopelia orientalis (Latham, 1790) & Oriental Turtle-dove & LC & $A, B$ \\
\hline 152 & Streptopelia tranquebarica (Hermann, 1804) & Red Collared-dove & LC & $A, B, G, M$ \\
\hline 153 & Streptopelia decaocto (Frivaldszky, 1838) & Eurasian Collared-dove & LC & $A, B, G, K, L, M$ \\
\hline 154 & Stigmatopelia senegalensis (Linnaeus, 1766) & Laughing Dove & LC & $A, B, G, K, L, M$ \\
\hline 155 & Stigmatopelia chinensis (Scopoli, 1786) & Spotted Dove & LC & $A, B, G, K, L, M$ \\
\hline \multirow[t]{2}{*}{156} & Treron phoenicopterus (Latham, 1790) & Yellow-footed Green-pigeon & LC & $\mathrm{B}, \mathrm{K}, \mathrm{L}, \mathrm{M}$ \\
\hline & Psittacidae & & & \\
\hline 157 & Psittacula eupatria (Linnaeus, 1766) & Alexandrine Parakeet & LC & $\mathrm{B}, \mathrm{K}, \mathrm{M}$ \\
\hline 158 & Psittacula krameri (Scopoli, 1769) & Rose-ringed Parakeet & LC & $A, B, G, H, K, L, M$ \\
\hline \multirow[t]{2}{*}{159} & Psittacula cyanocephala (Linnaeus, 1766) & Plum-headed Parakeet & LC & $A, B, G, K, L, M$ \\
\hline & Cuculidae & & & \\
\hline 160 & Clamator jacobinus (Boddaert, 1783) & Pied Cuckoo & LC & $\mathrm{B}, \mathrm{H}, \mathrm{M}$ \\
\hline 161 & Cuculus varius Vahl, 1797 & Common Hawk-cuckoo & LC & $\mathrm{A}, \mathrm{M}$ \\
\hline 162 & Cuculus canorus Linnaeus, 1758 & Common Cuckoo & LC & B \\
\hline
\end{tabular}




\begin{tabular}{|c|c|c|c|c|}
\hline & Species & Common Name & $\begin{array}{c}\text { IUCN Status } \\
2011\end{array}$ & References \\
\hline 163 & Eudynamys scolopaceus (Linnaeus, 1758) & Asian Koel & LC & $A, B, G, H, K, L, M$ \\
\hline 164 & Phaenicophaeus leschenaultii (Lesson, 1830) & Sirkeer Malkoha & LC & $\mathrm{A}, \mathrm{M}$ \\
\hline \multirow[t]{2}{*}{165} & Centropus sinensis (Stephens, 1815) & Greater Coucal & LC & $A, B, G, K, L, M$ \\
\hline & Tytonidae & & & \\
\hline \multirow[t]{2}{*}{166} & Tyto alba (Scopoli, 1769) & Barn Owl & LC & $\mathrm{A}, \mathrm{M}$ \\
\hline & Strigidae & & & \\
\hline 167 & Otus sunia (Hodgson, 1836) & Oriental Scops-owl & LC & B \\
\hline 168 & Otus bakkamoena Pennant, 1769 & Collared Scops-owl & LC & $A, B$ \\
\hline 169 & Bubo bengalensis (Franklin, 1831) & Rock Eagle-owl & LC & $A, B, G, K, M$ \\
\hline 170 & Bubo coromandus (Latham, 1790) & Dusky Eagle-owl & LC & M \\
\hline 171 & Ketupa zeylonensis (Gmelin, 1788) & Brown Fish-owl & LC & $\mathrm{A}, \mathrm{K}, \mathrm{M}$ \\
\hline 172 & Glaucidium radiatum (Tickell, 1833) & Jungle Owlet & LC & K \\
\hline 173 & Athene brama (Temminck, 1821) & Spotted Owlet & LC & $\mathrm{A}, \mathrm{B}, \mathrm{H}, \mathrm{K}, \mathrm{L}, \mathrm{M}$ \\
\hline 174 & Ninox scutulata (Raffles, 1822) & Brown Hawk-owl & LC & A \\
\hline \multirow[t]{2}{*}{175} & Asio flammeus (Pontoppidan, 1763) & Short-eared Owl & LC & M \\
\hline & Caprimulgidae & & & \\
\hline 176 & Caprimulgus indicus Latham, 1790 & Grey Nightjar & LC & $A, B$ \\
\hline 177 & Caprimulgus macrurus Horsfield, 1821 & Large-tailed Nightjar & LC & B \\
\hline 178 & Caprimulgus asiaticus Latham, 1790 & Indian Nightjar & LC & $A, G, L, M$ \\
\hline \multirow[t]{2}{*}{179} & Caprimulgus affinis Horsfield, 1821 & Savanna Nightjar & LC & B \\
\hline & Apodidae & & & \\
\hline 180 & Cypsiurus balasiensis (Gray, 1829) & Asian Palm-swift & LC & $\mathrm{A}, \mathrm{K}$ \\
\hline \multirow[t]{2}{*}{181} & Apus affinis (Gray, 1830) & House Swift & LC & $\mathrm{A}, \mathrm{B}, \mathrm{H}, \mathrm{L}, \mathrm{M}$ \\
\hline & Coraciidae & & & \\
\hline \multirow[t]{2}{*}{182} & Coracias benghalensis (Linnaeus, 1758) & Indian Roller & LC & $\mathrm{A}, \mathrm{B}, \mathrm{G}, \mathrm{H}, \mathrm{K}, \mathrm{L}, \mathrm{M}$ \\
\hline & Alcedinidae & & & \\
\hline 183 & Pelargopsis capensis (Linnaeus, 1766) & Stork-billed Kingfisher & LC & $\mathrm{B}, \mathrm{M}$ \\
\hline 184 & Halcyon smyrnensis (Linnaeus, 1758) & White-throated Kingfisher & LC & $\mathrm{A}, \mathrm{B}, \mathrm{G}, \mathrm{H}, \mathrm{K}, \mathrm{L}, \mathrm{M}$ \\
\hline 185 & Alcedo atthis (Linnaeus, 1758) & Common Kingfisher & LC & $\mathrm{A}, \mathrm{B}, \mathrm{G}, \mathrm{K}, \mathrm{L}, \mathrm{M}$ \\
\hline \multirow[t]{2}{*}{186} & Ceryle rudis (Linnaeus, 1758) & Pied Kingfisher & LC & $\mathrm{A}, \mathrm{B}, \mathrm{G}, \mathrm{H}, \mathrm{K}, \mathrm{L}, \mathrm{M}$ \\
\hline & Meropidae & & & \\
\hline 187 & Merops orientalis Latham, 1802 & Little Green Bee-eater & LC & $\mathrm{A}, \mathrm{B}, \mathrm{G}, \mathrm{L}, \mathrm{M}$ \\
\hline \multirow[t]{2}{*}{188} & Merops philippinus Linnaeus, 1766 & Blue-tailed Bee-eater & LC & $B, G, L, M$ \\
\hline & Upupidae & & & \\
\hline \multirow[t]{2}{*}{189} & Upupa epops Linnaeus, 1758 & Eurasian Hoopoe & LC & $\mathrm{A}, \mathrm{B}, \mathrm{H}, \mathrm{K}, \mathrm{M}$ \\
\hline & Bucerotidae & & & \\
\hline \multirow[t]{2}{*}{190} & Ocyceros birostris (Scopoli, 1786) & Indian Grey Hornbill & LC & $\mathrm{A}, \mathrm{B}, \mathrm{M}$ \\
\hline & Ramphastidae & & & \\
\hline 191 & Megalaima zeylanica (Gmelin, 1788) & Brown-headed Barbet & LC & $B$ \\
\hline \multirow[t]{2}{*}{192} & Megalaima haemacephala (Mller, 1776) & Coppersmith Barbet & LC & $\mathrm{B}, \mathrm{G}, \mathrm{K}, \mathrm{M}$ \\
\hline & Picidae & & & \\
\hline 193 & Jynx torquilla Linnaeus, 1758 & Eurasian Wryneck & LC & $\mathrm{K}, \mathrm{M}$ \\
\hline 194 & Dendrocopos nanus (Vigors, 1832) & Brown-capped Woodpecker & LC & B \\
\hline 195 & Dendrocopos mahrattensis (Latham, 1801) & Yellow-crowned Woodpecker & LC & $\mathrm{B}, \mathrm{K}, \mathrm{M}$ \\
\hline 196 & Picus xanthopygaeus (Gray \& Gray, 1846) & Streak-throated Woodpecker & LC & B \\
\hline 197 & Dinopium benghalense (Linnaeus, 1758) & Black-rumped Flameback & $\mathrm{LC}$ & $A, B, K, M$ \\
\hline 198 & Chrysocolaptes festivus (Boddaert, 1783) & White-naped Woodpecker & LC & B \\
\hline
\end{tabular}




\begin{tabular}{|c|c|c|c|c|}
\hline & Species & Common Name & $\begin{array}{c}\text { IUCN Status } \\
2011 \\
\end{array}$ & References \\
\hline & Campephagidae & & & \\
\hline 199 & Tephrodornis pondicerianus (Gmelin, 1789) & Common Woodshrike & LC & $A, B, M$ \\
\hline \multirow[t]{2}{*}{200} & Coracina macei (Lesson, 1831) & Large Cuckooshrike & LC & M \\
\hline & Aegithinidae & & & \\
\hline \multirow[t]{2}{*}{201} & Aegithina tiphia (Linnaeus, 1758) & Common lora & LC & $\mathrm{B}, \mathrm{K}, \mathrm{M}$ \\
\hline & Campephagidae & & & \\
\hline 202 & Pericrocotus cinnamomeus (Linnaeus, 1766) & Small Minivet & LC & $\mathrm{B}, \mathrm{G}, \mathrm{M}$ \\
\hline 203 & Pericrocotus erythropygius (Jerdon, 1840) & White-bellied Minivet & LC & B \\
\hline \multirow[t]{2}{*}{204} & Pericrocotus ethologus Bangs \& Phillips, 1914 & Long-tailed Minivet & LC & A \\
\hline & Laniidae & & & \\
\hline 205 & Lanius isabellinus Ehrenberg, 1833 & Rufous-tailed Shrike & LC & K \\
\hline 206 & Lanius cristatus Linnaeus, 1758 & Brown Shrike & LC & $A, K$ \\
\hline 207 & Lanius vittatus Valenciennes, 1826 & Bay-backed Shrike & LC & $A, B, G, K, M$ \\
\hline 208 & Lanius schach Linnaeus, 1758 & Long-tailed Shrike & LC & $A, B, G, K, M$ \\
\hline \multirow[t]{2}{*}{209} & Lanius excubitor Linnaeus, 1758 & Great Grey Shrike & LC & $\mathrm{B}, \mathrm{G}, \mathrm{K}, \mathrm{M}$ \\
\hline & Oriolidae & & & \\
\hline \multirow[t]{2}{*}{210} & Oriolus oriolus (Linnaeus, 1758) & Golden Oriole & LC & $\mathrm{B}, \mathrm{G}, \mathrm{L}, \mathrm{M}$ \\
\hline & Dicruridae & & & \\
\hline 211 & Dicrurus macrocercus Vieillot, 1817 & Black Drongo & LC & $A, B, G, K, L, M$ \\
\hline 212 & Dicrurus leucophaeus Vieillot, 1817 & Ashy Drongo & LC & $A, B$ \\
\hline \multirow[t]{2}{*}{213} & Dicrurus caerulescens (Linnaeus, 1758) & White-bellied Drongo & LC & $B, L, M$ \\
\hline & Rhipiduridae & & & \\
\hline \multirow[t]{2}{*}{214} & Rhipidura aureola Lesson, 1830 & White-browed Fantail & LC & L,M \\
\hline & Monarchidae & & & \\
\hline \multirow[t]{2}{*}{215} & Terpsiphone paradisi (Linnaeus, 1758) & Asian Paradise-flycatcher & LC & $B, L, M$ \\
\hline & Corvidae & & & \\
\hline 216 & Dendrocitta vagabunda (Latham, 1790) & Rufous Treepie & LC & $\mathrm{A}, \mathrm{H}, \mathrm{K}, \mathrm{L}, \mathrm{M}$ \\
\hline 217 & Corvus splendens Vieillot, 1817 & House Crow & LC & $\mathrm{A}, \mathrm{B}, \mathrm{G}, \mathrm{H}, \mathrm{K}, \mathrm{L}, \mathrm{M}$ \\
\hline 218 & Corvus macrorhynchos Wagler, 1827 & Large-billed Crow & LC & $A, B, G, K, L, M$ \\
\hline \multirow[t]{2}{*}{219} & Corvus corax Linnaeus, 1758 & Common Raven & LC & M \\
\hline & Paridae & & & \\
\hline \multirow[t]{2}{*}{220} & Parus major Linnaeus, 1758 & Great Tit & LC & $A, B, G, M$ \\
\hline & Hirundinidae & & & \\
\hline 221 & Riparia paludicola (Vieillot, 1817) & Plain Martin & LC & $A, B$ \\
\hline 222 & Hirundo concolor Sykes, 1832 & Dusky Crag-martin & LC & $A, K, L, M$ \\
\hline 223 & Hirundo rustica Linnaeus, 1758 & Barn Swallow & LC & $A, B, G, K, L, M$ \\
\hline 224 & Hirundo smithii Leach, 1818 & Wire-tailed Swallow & LC & $A, K, L$ \\
\hline 225 & Hirundo daurica Linnaeus, 1771 & Red-rumped Swallow & LC & $A, B, K, L, M$ \\
\hline \multirow[t]{2}{*}{226} & Hirundo fluvicola Blyth, 1855 & Streak-throated Swallow & LC & $\mathrm{A}, \mathrm{G}, \mathrm{K}, \mathrm{M}$ \\
\hline & Alaudidae & & & \\
\hline 227 & Mirafra cantillans Blyth, 1844 & Singing Bushlark & LC & L,M \\
\hline 228 & Mirafra assamica Horsfield, 1840 & Rufous-winged Lark & LC & A \\
\hline 229 & Mirafra erythroptera Blyth, 1845 & Indian Lark & LC & $A, G, L, M$ \\
\hline 230 & Ammomanes phoenicura (Franklin, 1831) & Rufous-tailed Lark & LC & $\mathrm{B}, \mathrm{G}, \mathrm{H}, \mathrm{M}$ \\
\hline 231 & Calandrella brachydactyla (Leisler, 1814) & Greater Short-toed Lark & LC & A \\
\hline 232 & Galerida cristata (Linnaeus, 1758) & Crested Lark & LC & $A, G, K, L, M$ \\
\hline 233 & Alauda gulgula Franklin, 1831 & Oriental Skylark & LC & $A, B$ \\
\hline 234 & Eremopterix griseus (Scopoli, 1786) & Ashy-crowned Sparrow-lark & LC & $A, B, G, H, K, L, M$ \\
\hline
\end{tabular}




\begin{tabular}{|c|c|c|c|c|}
\hline & Species & Common Name & $\begin{array}{c}\text { IUCN Status } \\
2011\end{array}$ & References \\
\hline & Cisticolidae & & & \\
\hline 235 & Cisticola juncidis (Rafinesque, 1810) & Zitting Cisticola & LC & B \\
\hline 236 & Prinia hodgsonii Blyth, 1844 & Grey-breasted Prinia & LC & A \\
\hline 237 & Prinia gracilis (Lichtenstein, 1823) & Graceful Prinia & LC & B \\
\hline 238 & Prinia sylvatica Jerdon, 1840 & Jungle Prinia & LC & K \\
\hline 239 & Prinia socialis Sykes, 1832 & Ashy Prinia & LC & $A, G, K, M$ \\
\hline \multirow[t]{2}{*}{240} & Prinia inornata Sykes, 1832 & Plain Prinia & LC & $\mathrm{G}, \mathrm{K}, \mathrm{M}$ \\
\hline & Pycnonotidae & & & \\
\hline 241 & Pycnonotus leucotis (Gould, 1836) & White-eared Bulbul & LC & $A, B, G, K, M$ \\
\hline \multirow[t]{2}{*}{242} & Pycnonotus cafer (Linnaeus, 1766) & Red-vented Bulbul & LC & $A, B, G, K, L, M$ \\
\hline & Sylviidae & & & \\
\hline 243 & Orthotomus sutorius (Pennant, 1769) & Common Tailorbird & LC & $\mathrm{B}, \mathrm{G}, \mathrm{K}, \mathrm{M}$ \\
\hline 244 & Acrocephalus stentoreus (Ehrenberg, 1833) & Clamorous Reed-warbler & LC & M \\
\hline 245 & Phylloscopus collybita (Vieillot, 1817) & Common Chiffchaff & LC & $L, M$ \\
\hline 246 & Phylloscopus griseolus Blyth, 1847 & Sulphur-bellied Warbler & LC & M \\
\hline 247 & Phylloscopus humei (Brooks, 1878) & Hume's Leaf-warbler & LC & B \\
\hline 248 & Hippolais rama (Sykes, 1832) & Sykes's Warbler & LC & A \\
\hline 249 & Sylvia curruca (Linnaeus, 1758) & Lesser Whitethroat & LC & $K, L, M$ \\
\hline \multirow[t]{2}{*}{250} & Sylvia hortensis (Gmelin,, 1789) & Orphean Warbler & LC & M \\
\hline & Timaliidae & & & \\
\hline 251 & Chrysomma sinense (Gmelin, 1789) & Yellow-eyed Babbler & LC & $L, M$ \\
\hline 252 & Turdoides caudata (Dumont, 1823) & Common Babbler & LC & $\mathrm{A}, \mathrm{B}, \mathrm{G}, \mathrm{K}, \mathrm{M}$ \\
\hline 253 & Turdoides malcolmi (Sykes, 1832) & Large Grey Babbler & LC & $A, G, K, L, M$ \\
\hline \multirow[t]{2}{*}{254} & Turdoides striata (Dumont, 1823) & Jungle Babbler & LC & $A, B, G, K, L, M$ \\
\hline & Zosteropidae & & & \\
\hline \multirow[t]{2}{*}{255} & Zosterops palpebrosus (Temminck, 1824) & Oriental White-eye & LC & $\mathrm{B}, \mathrm{G}, \mathrm{M}$ \\
\hline & Sturnidae & & & \\
\hline 256 & Acridotheres tristis (Linnaeus, 1766) & Common Myna & LC & $A, B, G, K, L, M$ \\
\hline 257 & Acridotheres ginginianus (Latham, 1790) & Bank Myna & LC & $A, B, G, K, L, M$ \\
\hline 258 & Sturnus pagodarum (Gmelin, 1789) & Brahminy Starling & LC & $\mathrm{A}, \mathrm{B}, \mathrm{G}, \mathrm{K}, \mathrm{M}$ \\
\hline 259 & Sturnus roseus (Linnaeus, 1758) & Rosy Starling & LC & $A, B, K, M$ \\
\hline 260 & Sturnus contra Linnaeus, 1758 & Asian Pied Starling & LC & $\mathrm{A}, \mathrm{B}, \mathrm{G}, \mathrm{K}, \mathrm{L}, \mathrm{M}$ \\
\hline \multirow[t]{2}{*}{261} & Sturnus vulgaris Linnaeus, 1758 & Common Starling & LC & B \\
\hline & Muscicapidae & & & \\
\hline 262 & Luscinia svecica (Linnaeus, 1758) & Bluethroat & LC & $\mathrm{K}, \mathrm{M}$ \\
\hline 263 & Copsychus saularis (Linnaeus, 1758) & Oriental Magpie Robin & LC & $A, B, G, K, M$ \\
\hline 264 & Saxicoloides fulicatus (Linnaeus, 1766) & Indian Robin & LC & $A, B, G, K, L, M$ \\
\hline 265 & Phoenicurus ochruros (Gmelin, 1774) & Black Redstart & LC & $\mathrm{A}, \mathrm{K}, \mathrm{L}, \mathrm{M}$ \\
\hline 266 & Saxicola torquatus (Linnaeus, 1766) & Common Stonechat & LC & $A, B, K, L, M$ \\
\hline 267 & Saxicola caprata (Linnaeus, 1766) & Pied Bushchat & LC & $A, B, K, L, M$ \\
\hline 268 & Oenanthe picata (Blyth, 1847) & Variable Wheatear & LC & $\mathrm{K}, \mathrm{L}$ \\
\hline 269 & Oenanthe deserti (Temminck, 1825) & Desert Wheatear & LC & K \\
\hline 270 & Oenanthe isabellina (Temminck, 1829) & Isabelline Wheatear & LC & $L, M$ \\
\hline 271 & Cercomela fusca (Blyth, 1851) & Indian Chat & LC & $\mathrm{A}, \mathrm{B}, \mathrm{G}, \mathrm{K}, \mathrm{L}, \mathrm{M}$ \\
\hline 272 & Monticola solitarius (Linnaeus, 1758) & Blue Rock Thrush & LC & $\mathrm{K}, \mathrm{M}$ \\
\hline 273 & Muscicapa dauurica Pallas, 1811 & Asian Brown Flycatcher & LC & $B, M$ \\
\hline 274 & Ficedula parva (Bechstein, 1792) & Red-breasted Flycatcher & LC & $L, M$ \\
\hline 275 & Ficedula superciliaris (Jerdon, 1840) & Ultramarine Flycatcher & LC & B \\
\hline
\end{tabular}




\begin{tabular}{|c|c|c|c|c|}
\hline & Species & Common Name & $\begin{array}{c}\text { IUCN Status } \\
2011\end{array}$ & References \\
\hline 276 & Eumyias thalassinus Swainson, 1838 & Verditer Flycatcher & LC & M \\
\hline 277 & Cyornis tickelliae Blyth, 1843 & Tickell's Blue-flycatcher & LC & $\mathrm{B}, \mathrm{M}$ \\
\hline \multirow[t]{2}{*}{278} & Culicicapa ceylonensis (Swainson, 1820) & Grey-headed Canary-flycatcher & LC & $L, M$ \\
\hline & Nectariniidae & & & \\
\hline \multirow[t]{2}{*}{279} & Nectarinia asiatica (Latham, 1790) & Purple Sunbird & LC & $A, B, G, K, L, M$ \\
\hline & Passeridae & & & \\
\hline 280 & Passer domesticus (Linnaeus, 1758) & House Sparrow & LC & $A, B, G, K, L, M$ \\
\hline 281 & Passer hispaniolensis (Temminck, 1820) & Spanish Sparrow & LC & M \\
\hline 282 & Passer montanus (Linnaeus, 1758) & Eurasian Tree Sparrow & LC & A \\
\hline \multirow[t]{2}{*}{283} & Petronia xanthocollis (Burton, 1838) & Chestnut-shouldered Petronia & LC & $A, G, L, M$ \\
\hline & Ploceidae & & & \\
\hline 284 & Ploceus benghalensis (Linnaeus, 1758) & Black-breasted Weaver & LC & B \\
\hline 285 & Ploceus manyar (Horsfield, 1821) & Streaked Weaver & LC & A \\
\hline \multirow[t]{2}{*}{286} & Ploceus philippinus (Linnaeus, 1766) & Baya Weaver & LC & A.B,G,K,M \\
\hline & Estrildidae & & & \\
\hline 287 & Amandava amandava (Linnaeus, 1758) & Red Avadavat & LC & M \\
\hline 288 & Amandava formosa (Latham, 1790) & Green Avadavat & VU & $\mathrm{N}$ \\
\hline 289 & Lonchura malabarica (Linnaeus, 1758) & White-throated Munia & LC & $A, K, L, M$ \\
\hline 290 & Lonchura punctulata (Linnaeus, 1758) & Scaly-breasted Munia & LC & $A, B$ \\
\hline \multirow[t]{2}{*}{291} & Lonchura malacca (Linnaeus, 1766) & Black-headed Munia & LC & $A, B$ \\
\hline & Motacilidae & & & \\
\hline 292 & Motacilla alba Linnaeus, 1758 & White Wagtail & LC & $\mathrm{A}, \mathrm{H}, \mathrm{L}, \mathrm{M}$ \\
\hline 293 & Motacilla madaraspatensis Gmelin, 1789 & White-browed Wagtail & LC & $A, B, G, H, K, L, M$ \\
\hline 294 & Motacilla citreola Pallas, 1776 & Citrine Wagtail & LC & K \\
\hline 295 & Motacilla flava Linnaeus, 1758 & Yellow Wagtail & LC & $A, B, G, K, M$ \\
\hline 296 & Motacilla cinerea Tunstall, 1771 & Grey Wagtail & LC & $A, B, G, K, L, M$ \\
\hline 297 & Anthus rufulus Vieillot, 1818 & Paddyfield Pipit & LC & $\mathrm{A}, \mathrm{B}, \mathrm{K}, \mathrm{L}, \mathrm{M}$ \\
\hline 298 & Anthus novaeseelandiae (Gmelin, 1789) & Australasian Pipit & LC & $\mathrm{H}$ \\
\hline 299 & Anthus campestris (Linnaeus, 1758) & Tawny Pipit & LC & B \\
\hline 300 & Anthus trivialis (Linnaeus, 1758) & Tree Pipit & LC & B \\
\hline 301 & Anthus hodgsoni Richmond, 1907 & Olive-backed Pipit & LC & M \\
\hline \multirow[t]{2}{*}{302} & Anthus spinoletta (Linnaeus, 1758) & Water Pipit & LC & $\mathrm{L}$ \\
\hline & Fringillidae & & & \\
\hline \multirow[t]{2}{*}{303} & Carpodacus erythrinus (Pallas, 1770) & Common Rosefinch & LC & $\mathrm{A}, \mathrm{K}$ \\
\hline & Emberizidae & & & \\
\hline 304 & Melophus lathami (Gray, 1831) & Crested Bunting & LC & B.G,M \\
\hline 305 & Emberiza stewarti (Blyth, 1854) & White-capped Bunting & LC & K \\
\hline 306 & Emberiza buchanani Blyth, 1844 & Grey-necked Bunting & LC & $\mathrm{K}, \mathrm{M}$ \\
\hline 307 & Emberiza melanocephala Scopoli, 1769 & Black-headed Bunting & LC & M \\
\hline 308 & Emberiza bruniceps Brandt, 1841 & Red-headed Bunting & LC & M \\
\hline
\end{tabular}

IUCN status: LC - Least Concern; NT - Near Threatened; VU - Vulnerable; EN - Endangered; CR - Critically Endangered.

References: A - This study (fieldwork in 2009 - 2010); B - This study (intermittent fieldwork between 2006 and 2008); C - BirdLife International (2012a); D - BirdLife International (2012b); E - Islam \& Rahmani (2002); F - Islam \& Rahmani (2004); G - Nair (2009); H - Sale (1982); I - Sharma et al. (1995); J - Sundar (2004); K -

Tigerwatch (2009); L - Vyas \& Singh (2004); M - Vyas et al. (in prep.); N - BirdLife International (2012c). 
Table 5. Checklist of mammals of the Chambal River Basin

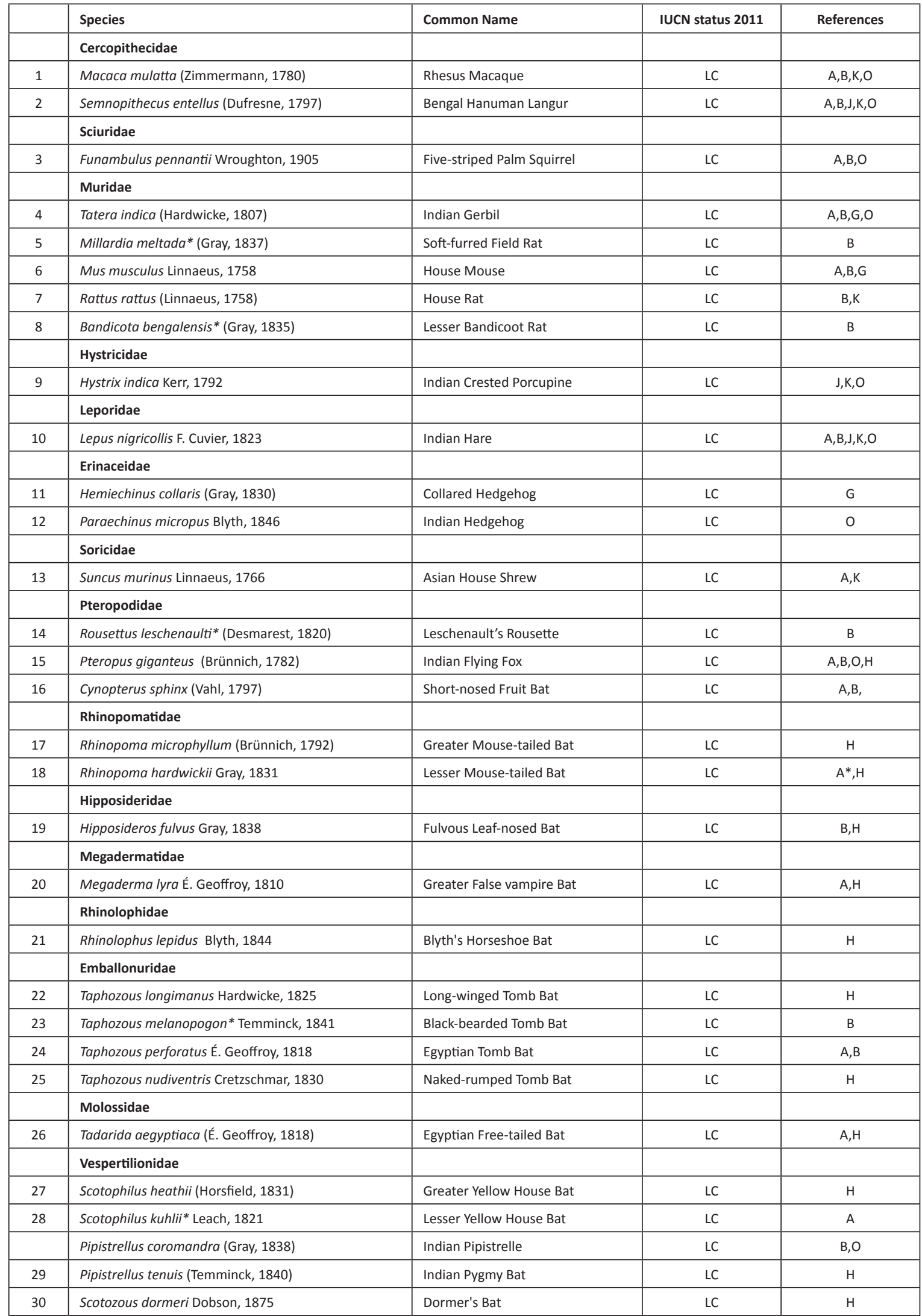




\begin{tabular}{|c|c|c|c|c|}
\hline & Species & Common Name & IUCN status 2011 & References \\
\hline & Manidae & & & \\
\hline \multirow[t]{2}{*}{31} & Manis crassicaudata É. Geoffroy, 1803 & Indian Pangolin & NT & C \\
\hline & Felidae & & & \\
\hline 32 & Felis chaus Schreber, 1777 & Jungle Cat & LC & $\mathrm{I}, \mathrm{K}, \mathrm{O}$ \\
\hline 33 & Felis silvestris Schreber, 1777 & Wild Cat / Desert Cat & LC & $\mathrm{E}, \mathrm{J}, \mathrm{O}$ \\
\hline 34 & Prionailurus bengalensis (Kerr, 1792) & Leopard Cat & LC & $\mathrm{K}, \mathrm{L}$ \\
\hline 35 & Prionailurus viverrinus Bennett, 1833 & Fishing Cat & EN & 0 \\
\hline 36 & Caracal caracal (Schreber, 1776) & Caracal & LC & $\mathrm{F}$ \\
\hline 37 & Panthera pardus (Linnaeus, 1758) & Leopard & NT & $\mathrm{K}, \mathrm{O}$ \\
\hline \multirow[t]{2}{*}{38} & Panthera tigris (Linnaeus, 1758) & Tiger & EN & K \\
\hline & Viverridae & & & \\
\hline 39 & Paradoxurus hermaphroditus (Pallas, 1777) & Asian Palm Civet & LC & $A, B, O$ \\
\hline \multirow[t]{2}{*}{40} & Viverricula indica (É. Geoffroy Saint-Hilaire, 1803) & Small Indian Civet & LC & $A, B, O$ \\
\hline & Herpestidae & & & \\
\hline 41 & Herpestes edwardsii (É. Geoffroy Saint-Hilaire, 1818) & Indian Grey Mongoose & LC & $A, B, K, O$ \\
\hline 42 & Herpestes javanicus (É. Geoffroy Saint-Hilaire, 1818) & Small Indian Mongoose & LC & $A, B, O$ \\
\hline \multirow[t]{2}{*}{43} & Herpestes smithii Gray, 1837 & Ruddy Mongoose & LC & $B, D$ \\
\hline & Hyaenidae & & & \\
\hline \multirow[t]{2}{*}{44} & Hyaena hyaena (Linnaeus, 1758) & Striped Hyaena & NT & $A, B, J, K, O$ \\
\hline & Canidae & & & \\
\hline 45 & Canis lupus pallipes Linnaeus, 1758 & Indian Wolf & LC & $\mathrm{J}, \mathrm{K}, \mathrm{O}$ \\
\hline 46 & Canis aureus Linnaeus, 1758 & Common Jackal & LC & $A, B, I, J, K, O$ \\
\hline 47 & Cuon alpinus (Pallas, 1811) & Dhole & EN & K \\
\hline 48 & Vulpes vulpes pusilla (Linnaeus, 1758) & Desert Fox & LC & K \\
\hline \multirow[t]{2}{*}{49} & Vulpes bengalensis (Shaw, 1800) & Indian Fox & LC & $\mathrm{J}, \mathrm{K}$ \\
\hline & Ursidae & & & \\
\hline \multirow[t]{2}{*}{50} & Melursus ursinus (Shaw, 1791) & Sloth Bear & VU & $\mathrm{K}, \mathrm{O}$ \\
\hline & Mustelidae & & & \\
\hline 51 & Mellivora capensis (Schreber, 1776) & Honey Badger & LC & K \\
\hline \multirow[t]{2}{*}{52} & Lutrogale perspicillata (I. Geoffroy Saint-Hilaire, 1826) & Smooth-coated Otter & VU & $\mathrm{A}, \mathrm{B}, \mathrm{M}, \mathrm{N}, \mathrm{J}, \mathrm{K}, \mathrm{O}$ \\
\hline & Suidae & & & \\
\hline \multirow[t]{2}{*}{53} & Sus scrofa cristatus Linnaeus, 1758 & Indian Wild Boar & LC & $\mathrm{A}, \mathrm{J}, \mathrm{K}, \mathrm{O}$ \\
\hline & Bovidae & & & \\
\hline 54 & Antilope cervicapra (Linnaeus, 1758) & Blackbuck & NT & $\mathrm{A}, \mathrm{K}, \mathrm{O}$ \\
\hline 55 & Gazella gazella (Pallas, 1766) & Chinkara & VU & $\mathrm{K}, \mathrm{O}$ \\
\hline 56 & Boselaphus tragocamelus (Pallas, 1766) & Nilgai & LC & $\mathrm{A}, \mathrm{J}, \mathrm{K}, \mathrm{O}$ \\
\hline \multirow[t]{2}{*}{57} & Tetracerus quadricornis (de Blainville, 1816) & Four-horned Antelope & VU & K \\
\hline & Cervidae & & & \\
\hline 58 & Axis axis (Erxleben, 1777) & Chital & LC & $\mathrm{J}, \mathrm{K}$ \\
\hline \multirow[t]{2}{*}{59} & Rusa unicolor (Kerr, 1792) & Sambar & VU & $A, J, K$ \\
\hline & Platanistidae & & & \\
\hline 60 & Platanista gangetica (Roxburgh, 1801) & Gangetic River Dolphin & EN & $\mathrm{A}, \mathrm{B}, \mathrm{M}, \mathrm{N}, \mathrm{I}, \mathrm{J}, \mathrm{K}, \mathrm{O}$ \\
\hline
\end{tabular}

IUCN status: LC - Least Concern; NT - Near Threatened; VU - Vulnerable; EN - Endangered; CR - Critically Endangered.

References: A - This study (fieldwork in 2009 - 2010); B - This study (intermittent fieldwork between 2006 and 2008); C - Chauhan \& Narain (2001); D - Choudhury et al. (2008); E - Driscoll \& Nowell (2009); F - Khudsar (2004); G - Molur et al. (2005); H - Molur et al. (2002); I - Nair (2009); J - Rao (1988); K - Sale (1982); L - Sanderson et al. (2008); M - Sharma et al. (1995); N - Vyas (2004); O - Vyas et al. (in prep.)

* Identification based on comparison of field observations with standard identification / field guides. Individuals were not available for anatomical or morphological examination. This record needs to be verified. 
Hindu 2006a, b).

\section{CONCLUSION}

Our effort is intended at providing a peer-reviewed and open-access compilation of vertebrate fauna of the Chambal River Basin, which highlights the region's ecological significance. We believe that this checklist will serve as a baseline for assessing changes in species status, distributions and occurrences in the face of threats; inform protected area managers, conservationists and environment impact assessors; and serve as a platform to initiate participatory biodiversity monitoring initiatives.

\section{REFERENCES}

Abraham, R. (2011). Puntius fasciatus. In: IUCN 2012. IUCN Red List of Threatened Species. Version 2012.1. <www.iucnredlist.org>. Downloaded on 20 August 2012.

Abraham, R.K., N. Kelkar \& A.B. Kumar (2011). Freshwater fish fauna of the Ashambu Hills landscape, southern Western Ghats, India with notes on some range extensions. Journal of Threatened Taxa 3(3): 1585-1593.

Bennett, D., M. Gaulke, E.R. Pianka, R. Somaweera \& S.S. Sweet (2010). Varanus salvator. In: IUCN 2011. IUCN Red List of Threatened Species. Version 2011.2. <www.iucnredlist.org>. Downloaded on 05 June 2012.

BirdLife International (2012a). Important Bird Areas factsheet: National Chambal Wildlife Sanctuary (Agra/Etawah) (part of National Chambal River Gharial Sanctuary AZE). Downloaded from http://www.birdlife.org on 06/05/2012.

BirdLife International (2012b). Important Bird Areas factsheet: National Chambal Wildlife Sanctuary (Bundi/Kota) (part of National Chambal River Gharial Sanctuary AZE). Downloaded from http:// www.birdlife.org on 06/05/2012.

BirdLife International (2012c). Species factsheet: Amandava formosa Downloaded from http://www.birdlife.org on 05/05/2012.

BirdLife International (2012). IUCN Red List for Birds. Downloaded from http://www.birdlife.org on 06/05/2012.

Böhm, M. \& N. Richman (2010). Ophisops microlepis. In: IUCN 2011. IUCN Red List of Threatened Species. Version 2011.2. <www. iucnredlist.org>. Downloaded on 27 April 2012.

Champion, H.G. \& S.K. Seth (1968). A Revised Survey of the Forest Types of India. Manager of Publication, New Delhi, 404pp.

Chandra, K. \& P.U. Gajbe (2005). An inventory of Herpetofauna of Madhya Pradesh and Chhattisgarh. Zoos' Print Journal 20(3): 18121819.

Chauhan, R. \& S. Narain (2001). The Indian Pangolin (Manis crassicaudata) in the Chambal ravines of Etawah. Zoos' Print Journal 16(5): 501.

Choudhury, A., C. Wozencraft, D. Muddapa \& P. Yonzon (2008). Herpestes smithii. In: IUCN 2010. 2010 IUCN Red List of Threatened Species. <www.iucnredlist.org>. Downloaded on 06 September 2010.

Das, I., D. Basu \& S. Singh (2010). Nilssonia hurum (Gray 1830) - Indian peacock softshell turtle. In: Rhodin, A.G.J., P.C.H. Pritchard, P.P. van Dijk, R.A. Saumure, K.A. Buhlmann, J.B. Iverson \& R.A. Mittermeier (eds.). Conservation Biology of Freshwater Turtles and Tortoises: A Compilation Project of the IUCN/SSC Tortoise and Freshwater Turtle Specialist Group. Chelonian Research Monographs No. 5. 6pp.
Department of Water Resources (Government of Rajasthan). Chambal Basin. Accessed on 02.11.12. (http://waterresources.rajasthan.gov. in/3bchambal.htm).

Driscoll, C. \& K. Nowell (2009). Felis silvestris. In: IUCN 2010. 2010 IUCN Red List of Threatened Species. <www.iucnredlist.org>. Downloaded on 06 September 2010.

Dubey, G.P. \& R.K. Mehra (1959). Fish and fisheries of Chambal River. Proceedings of the All India Congress of Zoology 1: 647-665.

Eschmeyer, W.N. (ed.) (2012). Catalog of Fishes. California Academy of Sciences. Electronic version accessed on 23.04.2012 <http:// research.calacademy.org/research/ichthyology/catalog/ fishcatmain.asp>

Eschmeyer, W.N. \& J.D. Fong (2012). Species of Fishes by family/ subfamily. On-line version dated 23.04.2012. http://research. calacademy.org/research/ichthyology/catalog/SpeciesByFamily.asp

Gopal, L. \& V.C. Srivastava (2008). History of Agriculture in India (up to c. 1200 A.D.). History of Science, Philosophy and Culture in Indian Civilization. Project of History of Indian Science, Philosophy and Culture. Centre for Studies in Civilizations, New Delhi, xxxiv+912pp.

Hussain, S.A. (1991). Ecology of Gharial (Gavialis gangeticus) in National Chambal Sanctuary. MPhil Dissertation. Centre for Wildlife and Ornithology. Aligarh Muslim University, 57pp.

Hussain, S.A. (1999). Reproductive success, hatchling survival and rate of increase of gharial Gavialis gangeticus in National Chambal Sanctuary, India. Biological Conservation 87(2): 261-268.

Hussain, S.A. (2009). Basking site and water depth selection by gharial Gavialis gangeticus Gmelin, 1789 (Crocodylia, Reptilia) in National Chambal Sanctuary, India and its implication for river conservation. Aquatic Conservation-Marine and Freshwater Ecosystems 19(2): 127-133.

Hussain, S.A. \& R. Badola (2001). Integrated conservation planning for Chambal River Basin. Paper presented in the National Workshop on Regional Planning for Wildlife Protected Areas. August 6-8, 2001. India Habitat Centre, New Delhi, Wildlife Institute of India, Dehra Dun, 20pp.

Islam, M.Z. \& A.R. Rahmani (2002). Threatened birds of India. Buceros 7(1\&2). Compiled from Threatened Birds of Asia - BirdLife International Red Data Book (2001). BirdLife International, Cambridge, U.K.

Islam, M.Z. \& A.R. Rahmani (2004). Important Bird Areas in India: Priority Sites for Conservation. Indian Bird Conservation Network: Bombay Natural History Society and BirdLife International (UK), xviii+1133pp.

ITIS (2012). Integrated Taxonomic Information System, online database available at http://www.itis.gov. Viewed on 05 May 2012.

IUCN (2011). IUCN Red List of Threatened Species. Version 2011.2. <www.iucnredlist.org>. Downloaded on 23 April 2012.

Jain, S.K., P.K. Agarwal \& V.P. Singh (2007). Hydrology and Water Resources of India - Volume 57 of Water Science and Technology Library - Tributaries of Yamuna River. Springer, 350pp.

Knight, J.D.M., K.R. Devi \& V. Atkore (2011). Systematic status of Systomus rubrotinctus Jerdon (Teleostei: Cyprinidae) with notes on the Puntius arulius group of fishes. Journal of Threatened Taxa 3(4): 1686-1693.

Katdare, S., A. Srivathsa, A. Joshi, P. Panke, R. Pande, D. Khandal \& M. Everard (2011). Gharial (Gavialis gangeticus) populations and human influences on habitat on the River Chambal, India. Aquatic Conservation: Marine and Freshwater Ecosystems 21(4): 364-371.

Kaul, O.N. (1962). Management of Chambal ravines in Rajasthan. Indian Forester 88(10): 725-730.

Khudsar, F.A. (2004). Sighting of caracal in the Chambal ravines of Bhind District, Madhya Pradesh. Journal of the Bombay Natural History Society 101(1): 149.

MoEF (2011). Ministry of Environment and Forests - Wildlife Division. Summary records of the $22^{\text {nd }}$ Meeting of the Standing Committee of National Board for Wildlife (NBWL) held on 25 th April 2011 in Paryavaran Bhavan, CGO Complex, Lodi Road, New Delhi-110003. Available at http://moef.nic.in/divisions/wildlife/NBWL-22-Mom. pdf 
Moll, E.O. (1997). Effects of habitat alteration on river turtles of tropical Asia with emphasis on sand mining and dams, pp. 37-41. In Abbema, J.V. (ed.). Proceedings: Conservation, Restoration, and Management of Tortoises and Turtles - An International Conference, New York. New York Turtle and Tortoise Society.

Molur, S. \& S. Walker (1998a). Report of the Workshop "Conservation Assessment and Management Plan for Freshwater Fishes of India". Zoo Outreach Organisation \& CBSG South Asia, Coimbatore, India, 156 p.

Molur S. \& S. Walker (eds.) (1998b). Reptiles of India. Report on Biodiversity Conservation Prioritization Project (BCPP) India Endangered Species Project, Conservation Assessment and Management Plan Workshop. Zoo Outreach Organisation \& CBSG South Asia, Coimbatore, India, 175pp.

Molur, S., C. Srinivasulu, B. Srinivasulu, S. Walker, P.O. Nameer \& L. Ravikumar (2005). Status of South Asian Non-volant Small Mammals: Conservation Assessment and Management Plan Workshop Report. Zoo Outreach Organisation \& CBSG-South Asia, Coimbatore, India, 618pp.

Molur, S., G. Marimuthu, C. Srinivasulu, S. Mistry, A.M. Hutson, P.J.J. Bates, S. Walker, K. Padma Priya \& A.R. Binu Priya (eds.) (2002) Status of South Asian Chiroptera: Conservation Assessment and Management Plan Workshop Report. Zoo Outreach Organisation, CBSG South Asia \& WILD, Coimbatore, India, viii+320pp.

Murphy, J. \& A. Lobo (2010). Enhydris sieboldii. In: IUCN 2011. IUCN Red List of Threatened Species. Version 2011.2. <www.iucnredlist. org>. Downloaded on 27 April 2012.

Murthy, R.S. (2004). Management Plan of National Chambal Sanctuary, Morena (M.P.) 2003-2004 to 2013-2014. Forest Department, Government of Madhya Pradesh, Bhopal, India.

Nair, A.K. (2009). The status and distribution of major aquatic fauna in the National Chambal Gharial Sanctuary in Rajasthan with special reference to the Gangetic Dolphin Platanista gangetica gangetica (Cetartiodactyla: Platanistidae). Journal of Threatened Taxa 1(3): 141-146.

Nair, T. (2010). Ecological and anthropogenic covariates influencing gharial Gavialis gangeticus distribution and habitat use in Chambal River, India. Unpublished Master's Thesis. National Centre for Biological Sciences, Tata Institute of Fundamental Research, iv $+74 p p$.

Ng, H.H. \& M. Kottelat (2008). The identity of Clarias batrachus (Linnaeus, 1758), with the designation of a neotype (Teleostei: Clariidae). Zoological Journal of the Linnean Society 153: 725-732.

Nilsson, C., C.A. Reidy, M. Dynesius \& C. Revenga (2005). Fragmentation and flow regulation of the world's large river systems. Science 308 405-408.

NWDA. Parbati Kalisindh Chambal Link. National Water Development Agency. Ministry of Water Resources, Government of India. Available at http://www.nwda.gov.in/index4.asp?ssslid=125\&subsubsublinki $\mathrm{d}=45$ \&langid=1. Accessed on 10 May 2012.

Pethiyagoda, R., M. Meegaskumbura \& K. Maduwage (2012). A synopsis of the South Asian fishes referred to Puntius (Pisces: Cyprinidae). Ichthyological Exploration of Freshwaters 23(1): 6995.

Prasad, R. (1988). Effectiveness of aerial seeding in reclamation of Chambal ravines in Madhya Pradesh. Indian Forester 114(1): 1-18.

Rao, R.J. (1988). Nesting Ecology of Gharial in the National Chambal Sanctuary. Wildlife Institute of India, Dehradun, India, 105pp.

Reddy, P.A., D.S. Gour, M. Bhavanishankar, K. Jaggi, S.M. Hussain, K. Harika \& S. Shivaji (2012). Genetic evidence of tiger population structure and migration within an isolated and fragmented landscape in northwest India. PLOS ONE 7(1): e29827. doi:10.1371/ journal.pone.0029827.

RUSDIP (2008). Environmental Assessment Document. Initial Environmental Examination: Dholpur Water Supply Subproject. Project Number: 40031. September 2008. India: Rajasthan Urban Sector Development Investment Program. Prepared by Local Self Government Department For the Government of Rajasthan, Rajasthan Urban Infrastructure Development Project. Available at
<http://www.ruidp.gov.in/work-progress/IEE\%20PDF/Water\%20 Supply\%20Project\%20of\%20Dholpur.pdf>

Saksena, D.N. (2007). Fish diversity of Northern Madhya Pradesh (Gwalior and Chambal Divisions), pp. 50-57. In: Lakra, W.S. \& U.K Sarkar (eds.). Fresh Water Fish Diversity of Central India. National Bureau of Fish Genetic Resources, Indian Council of Agricultural Research, xiv+183pp.

Sale, J.B. (1982). 2nd Draft. Management Plan For The National Chambal Sanctuary. First Five Year Period 1982/83 - 1986/87. Central Crocodile Breeding and Management Institute, Hyderabad, iii+82pp.

Sanderson, J., S. Sunarto, A. Wilting, C. Driscoll, R. Lorica, J. Ross, A. Hearn, S. Mukherjee, J.A. Khan, B. Habib \& L. Grassman (2008). Prionailurus bengalensis. In: IUCN 2010. 2010 IUCN Red List of Threatened Species. <www.iucnredlist.org >.

Schätti, B. \& A. Schmitz (2006). Re-assessing Platyceps ventromaculatus (Gray, 1834) (Reptilia: Squamata: Colubrinae). Revue suisse de Zoologie 113(4): 747-768.

Sharma, L.L. \& C.S. Choudhary (2007). Conservation and management of fish diversity in Rajasthan, pp. 110-117. In: Lakra, W.S. \& U.K. Sarkar (eds.). Fresh Water Fish Diversity of Central India. National Bureau of Fish Genetic Resources, Indian Council of Agricultural Research, xiv+183pp.

Sharma, R.K., R. Mathur \& S. Sharma (1995). Status and distribution of fauna in National Chambal Sanctuary, Madhya Pradesh. Indian Forester 121(10): 912-916.

Sharma, S.K. (2003). Presence of Dumeril's Black-headed Snake (Sibynophis subpunctatus) in Kumbhalgarh Wildlife Sanctuary, Rajasthan, India. Cobra 53: 17-18.

Sivakumar, K. (2002). The rare freshwater giant stingray in the National Chambal Sanctuary: Needs more attention for conservation. WII Newsletter 8(4) \& 9(1): 5.

Sivakumar, K. \& B.C. Choudhury (2008). Chambal River, Rajasthan: Importance of water flow and minimum water level in conservation of all tropic levels in different habitats and biodiversity. Journal of Landscape Architecture 19: 52-57.

Srivastava, N. (2007). Freshwater fish diversity in Rajasthan, pp 142155. In: Lakra, W.S. \& U.K. Sarkar (eds.). Fresh Water Fish Diversity of Central India. National Bureau of Fish Genetic Resources, Indian Council of Agricultural Research, xiv+183pp.

Sundar, G. (2004). Observations on breeding Indian Skimmers Rynchops albicollis in the National Chambal Sanctuary, Uttar Pradesh, India. Forktail 20: 89-90.

Taigor, S.R. \& R.J. Rao (2010). Anthropogenic threats in the National Chambal Sanctuary, Madhya Pradesh, India. Tigerpaper 37(1): 2327.

Tenzin, K. (2010). Gibelion catla. In: IUCN 2012. IUCN Red List of Threatened Species. Version 2012.1. <www.iucnredlist.org>. Downloaded on 14 August 2012.

The Hindu (2006a). Illegal sand mining a threat to the Chambal river: M.P. Minister. 22 July 2006, The Hindu. Online edition available at http://www.hindu.com/2006/07/22/stories/2006072215220300. htm

The Hindu (2006b). Illegal sand mining threatens Gharial sanctuary. 15 October 2006, The Hindu. Online edition available at http://www. hindu.com/2006/10/15/stories/2006101511900500.htm

Tigerwatch (2008). Chambal expedition - January '08 report. Tigerwatch, Ranthambore, India, 64pp.

Tigerwatch (2009). The Gharial expedition - December 2009 - A survey of Gharial populations in one segment of the National Chambal Sanctuary, Rajasthan. Tigerwatch, Ranthambore, India, 37pp.

Turtle Taxonomy Working Group [van Dijk, P.P., J.B. Iverson, H.B. Shaffer, R. Bour \& A.G.J. Rhodin] (2011). Turtles of the world, 2011 update: annotated checklist of taxonomy, synonymy, distribution, and conservation status, pp. 165-242. In: Rhodin, A.G.J., P.C.H. Pritchard, P.P. van Dijk, R.A. Saumure, K.A. Buhlmann, J.B. Iverson \& R.A. Mittermeier (eds.). Conservation Biology of Freshwater Turtles and Tortoises: A Compilation Project of the IUCN/SSC Tortoise and Freshwater Turtle Specialist Group. Chelonian Research Monographs 
No. 5., 237pp

Vidthayanon, C., I. Baird \& Z. Hogan (2011). Himantura polylepis. In: IUCN 2011. IUCN Red List of Threatened Species. Version 2011.2. <www.iucnredlist.org>. Downloaded on 23 April 2012.

Vishwanath, W. (2010a). Clarias magur. In: IUCN 2012. IUCN Red List of Threatened Species. Version 2012.1. <www.iucnredlist.org>. Downloaded on 14 August 2012.

Vishwanath, W. (2010b). Megarasbora elanga. In: IUCN 2012. IUCN Red List of Threatened Species. Version 2012.1. <www.iucnredlist. org>. Downloaded on 14 August 2012.

Vyas, R. (2004). Fishing in protected areas-a bane for aquatic wildlife. Zoos' Print XIX(7): 1-2.
Vyas, R. \& H. Singh. (2004). Biodiversity survey of Gandhi Sagar Reservoir, Madhya Pradesh. Zoos' Print Journal 19(7): 1525-1529.

Vyas, R., R.S. Tomar \& S. Singhal (in prep.) Macrofauna of National Chambal Sanctuary in Rajasthan and its conservation issues.

Whitaker, R. \& Z. Whitaker (1980). Distribution and status of Varanus salvator in India and Sri Lanka. Herpetological Review 11(3): 81-82.

Wilson, D.E. \& D.M. Reeder (eds.) (2005). Mammal Species of the World: A Taxonomic and Geographic Reference, $3^{\text {rd }}$ edition, The Johns Hopkins University Press, Baltimore, xvii+2142pp. 